\title{
Design and Control of a Novel Variable Stiffness Soft arm
}

\section{Lina $\mathrm{Hao}^{1,4}$ Chaoqun Xiang ${ }^{1,2,3}$ M.E. Giannaccini2 ${ }^{2,3}$ Hongtai Cheng ${ }^{1}$ Ying Zhang ${ }^{1}$}

\author{
S. Nefti-Meziani ${ }^{2}$ Steven Davis ${ }^{2}$ \\ (1. School of Mechanical Engineering \& Automation, Northeastern University, Shenyang 110819, China \\ 2. Centre for Autonomous Systems and Advanced Robotics, University of Salford, Salford, United Kingdom \\ 3. Soft Robotics Group, Bristol Robotics Laboratory, University of Bristol, Bristol, United Kingdom \\ 4.haolina@me.neu.edu.cn)
}

\begin{abstract}
Soft robot arms possess such characteristics as light weight, simple structure and good adaptability to the environment, among others. On the other hand, robust control of soft robot arms presents many difficulties. Based on these reasons, this paper presents a novel design and modelling of a fuzzy active disturbance rejection control (FADRC) controller for a soft PAM arm. The soft arm comprises three contractile and one extensor PAMs, which can vary its stiffness independently of its position in space. Force analysis for the soft arm is conducted, and stiffness model of the arm is established based on the relational model of contractile and extensor PAM. The accuracy of stiffness model for the soft arm was verified through experiments. Associated to this, a controller based on the fuzzy adaptive theory and ADRC, FADRC, has been designed to control the arm. The fuzzy adaptive theory is used to adjust the parameters of the ADRC, the control algorithm has the ability to control stiffness and position of the soft arm. In this paper, FADRC was further verified through comparative experiments on the soft arm. This paper reinforces the hypothesis that FADRC control, as an algorithm, indeed possesses good robustness and adaptive abilities.
\end{abstract}

Key words: soft robot, variable stiffness, PAM, stiffness modelling, FADRC

\section{Introduction}

The rapid development of robot technology, entails that rigid robots enjoy a very wide range of applications. Conventional robots essentially mimic mammals, which possesses rigid links ${ }^{[1]}$. Due to the limited degrees of freedom (DOF) and main component materials of rigid robot, they also suffer from some disadvantages such as high cost, heavy weight and poor compatibility with unstructured environments. Soft robots enjoy low cost, light weight, softness, simple structure among other advantages. Soft and compliant robots are particularly apt for unstructured environments due to their flexibility, versatility and claims to safety.

In general, actuation strategies for soft robots are chosen in order not to interfere with the compliance of their body. For example, the actuation can be achieved pneumatically or with cables and thin shape memory alloy wires ${ }^{[2]}$. For instance, OctArm ${ }^{[3]}$ and continuum manipulators ${ }^{[1]}$ are examples of pneumatic soft arms. The design principle of the OctArm is bioinspired. It imitates hydrostatic skeletons such as the elephant trunk and the octopus tentacles. These impressive animal structures are completely boneless, but nevertheless can produce bending, contraction, and elongation movements ${ }^{[4]}$. This kind of robot possesses the advantage of light weight, flexibility and increased claims to safety compared to conventional robots. Chen, developed one section of a pneumatic continuum robot (Clobot) ${ }^{[5]}$ that is made of silicone rubber. It can bend $120^{\circ}$ under $0.2 \mathrm{mbar}$. Another pneumatic and continuum arm, the Bionic Handling Assistant (BHA) ${ }^{[6]}$, was designed by the Festo Company. . Although the resultant soft robots are lightweight and flexible, their bodies still retain much rigidity, which is used to maintain their shapes. Hence their stiffness does not change with change in position and load.

However, lightweight, high flexibility and variable stiffness still remain the most valid solutions for increasing safety when considering physical human-robot cooperation ${ }^{[7]}$. Based on further research results, a variable stiffness structure was able to switch between resisting components and comply to external forces ${ }^{[8]}$. In this way, the robot would be compliant when the task required it. This is essential for safe human-robot interactions, though the soft robot would again become stiff for purposes of precise positioning. The first variable stiffness method is the active stiffness control, and an example of this approach is LWRIII ${ }^{[9]}$. The other variable stiffness method for robot is usting variable stiffness/impedance actuators, and an example of this approach is the work of Tonietti ${ }^{[10]}$. In reference ${ }^{[11]}$ and ${ }^{[10]}$, they use variable stiffness actuator, and can change the stiffness of the robot without changing the position. These robots can behave in a soft manner these robots could be seen as 'soft' robots. However, the variable stiffness actuators robots are made with rigid parts and thus intrinsically different from the robot in ${ }^{[12]}$ and ${ }^{[13]}$, which can be physically soft. The materials of ${ }^{[12]}$ and ${ }^{[13]}$, robots allow a soft physical structure, and this robot can be named soft robot ${ }^{[14]}$. Normally, the actuator can form the whole soft robot. This can be achieved by filling selected items with air or water. The authors of ${ }^{[13,15,16]}$ chose to fill selected items with different solid particles to realize variable stiffness. In reference ${ }^{[17,18]}$, the authors used hydraulics to attain variable stiffness operations. However, high bulk modulus fluids have a disadvantage. These will increase the weight of the structure considerably. This becomes a 
problem unless high-bulk modulus fluids are used in underwater soft robots ${ }^{[19]}$. To avoid excessive weight, this paper proposes a variable stiffness soft arm driven by PAMs. A PAM is a two layered system consisting of an inner elastomeric bladder surrounded by an external woven braided shell. The wind angle of the braided shell, $\varphi$, is of critical importance. If $\varphi>54^{\circ} 44^{\prime}$, then the PAM is an expanding muscle and the muscle elongates when it is pressurized. But if $\varphi<54^{\circ} 44^{\prime}$, then the PAM is a contractor muscle ${ }^{[3,20]}$ and the muscle contracts when pressurized. Soft robots made of PAMs comply with the requirements for an intrinsically safer physical human-robot interaction. Michael B. Pritts, and et al. have also developed a continuum robot driven by contractile and one extensor PAMs, but the stiffness of the arm can not be varied independently from their end-effector position in space ${ }^{[21]}$. Koichi Suzumori, and et al. also have develop a continuum robot driven by contractile and one extensor PAMs, however the structure of arm is one contractile PAM at the center of the arm and the five extensor PAMs are equidistant from the center of the arm, under the same size, normally the output force of contractile PAM is larger extensor PAM, so the structure will limit the output force of the arm ${ }^{[22]}$.

PAMs possesses very strong nonlinearity, which is not convenient for control. Active disturbance rejection control (ADRC) is proposed by Han ${ }^{[23]}$, and possesses properties of active disturbance rejection which estimate disturbance and compensation in real time. Furthermore an ADRC controller does not need an accurate model system, and possesses the properties of active disturbance rejection, which refers to the function of estimating disturbance and compensation in real time ${ }^{[23,24]}$. ADRC has been employed in the domain of nonlinear control system of robot driven by PAMs, such as a PAM mechanism to achieve angle tracking precisely under varying load conditions ${ }^{[25]}$, positioning control of the one-DOF manipulator driven by pneumatic artificial muscles ${ }^{[26]}$, and trajectory tracking and decoupling for a two-joint system driven by PAMs ${ }^{[27]}$. However, too many parameters of ADRC need to be adjusted, which is not convenient to use. In the reference ${ }^{[28]},{ }^{[29]}$ and ${ }^{[30]}$, a fuzzy adaptive theory is used to adjust the parameters of ADRC, which achieved a good result. Thus Fuzzy adaptive theory is employed to adjust the parameters of ADRC in the paper.

In this paper, a novel soft arm made of one expansive PAM and three contractile PAMs, described in ${ }^{[31]}$ and that uses the principle of decoupled variable stiffness and positioning is utilised. Due to the ability to vary its stiffness, when the arm comes across a disturbance force, it will increase stiffness. This will decrease deformation and increase its control accuracy and robustness. If a human collides with the arm, the arm can decrease its stiffness and become softer and safer for the human. Both its stiffness model and kinematics model have been established based on the working theory of the arm. In this paper, the FADRC controller is proposed to take advantage of its adaptive properties as described within the fuzzy adaptive theory. Verification of the design controller was completed in experiments based on the variable stiffness of the soft arm. The experimental results demonstrated better accuracy and stability than would be obtained from testing results with the traditional PID controller.

The paper is structured as follows: in the first section, the structure and working principle of the arm are introduced. In the second and third sections, the kinematics model and the stiffness model of the arm are established, respectively. In the following sections, FADRC is proposed, and position and stiffness of the arm are controlled through this controller. General conclusions relating to the paper are formulated in Section seven.

\section{Description of variable stiffness soft arm}

\subsection{Design concept}

The novel variable stiffness soft arm utilised in this paper comprises the design of four PAMs. These are denoted as one central extensor PAM and three contractile PAMs. The extensor muscle sits at the center of the arm. The three contractile muscles are equidistant from the center of the arm, $\left(35 \mathrm{~mm}\right.$ away). These are placed at $120^{\circ}$ from each other. All actuators are secured to $150 \mathrm{~mm}$ diameter mounting plates at both ends of the arm. Once pressurized, contractor actuators contract by about $25 \%$ and the extensor almost doubles in length. But within the same geometric size, the output force of contractor actuators is much higher than extensor PAM. The placement of contractor actuators on the outside of the arm is to increase the possible payload. The higher the difference in length between the two kinds of PAMs, the bigger the working range that the arm can achieve. Hence, soft robot arms that have extensor muscles in their structure are able to reach higher curvatures than those that employ only contractile muscles. In reference ${ }^{[32]}$, a PAM was designed and mounted in the middle of the arm. However, its motion was derived from a motor and this lowered the levels of its flexibility, load-carrying capacity and its rigidity. In this paper the design includes ties that are utilized in order to ensure the actuators remain equidistant apart along the length of the arm. The ties ensure flexibility and were made of flexible nylon. The nylon ties also ensure that all the contractile PAMs are always in contact with the extensor PAM. The ties in the manipulator are located approximately every $25 \mathrm{~mm}$ along the length of the actuator. The structure of the soft arm is shown in figure 1 and the main structure size of the arm is shown in table 1. 


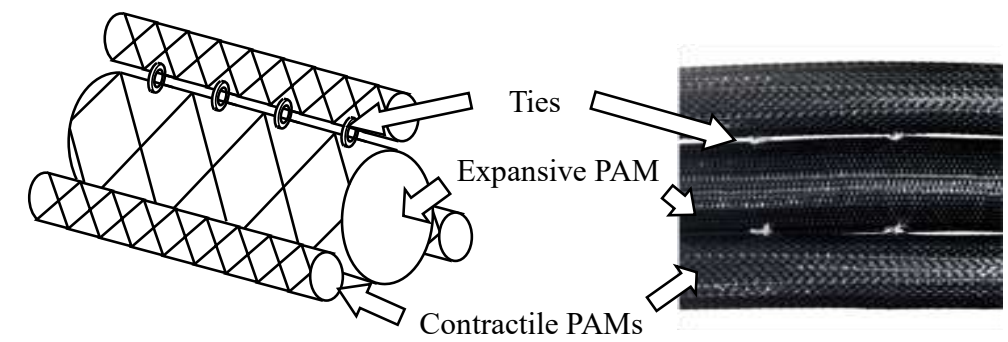

Fig.1 Schematics of the arm

Tab.1 The geometric parameters of arm and PAMs

\begin{tabular}{|c|c|c|c|c|c|c|c|c|}
\hline \multirow[b]{2}{*}{$\begin{array}{l}\text { Length of the arm(no } \\
\text { pressurized) } \\
\text { /mm }\end{array}$} & \multicolumn{4}{|c|}{ Expansive PAM } & \multicolumn{4}{|c|}{ Contractile PAM } \\
\hline & $\begin{array}{l}\text { Initial length } \\
\qquad / \mathrm{mm}\end{array}$ & $\begin{array}{c}\text { Initial } \\
\text { diameter } / \mathrm{mm}\end{array}$ & $\begin{array}{c}\text { Initial braid } \\
\text { angle }^{\circ}\end{array}$ & $\begin{array}{l}\text { Maximum } \\
\text { inflation } \\
\text { pressure } \\
\text { /bar }\end{array}$ & $\begin{array}{l}\text { Initial length } \\
\qquad / \mathrm{mm}\end{array}$ & $\begin{array}{c}\text { Initial } \\
\text { diameter } / \mathrm{mm}\end{array}$ & $\begin{array}{c}\text { Initial braid } \\
\text { angle }^{\circ}\end{array}$ & $\begin{array}{c}\text { Maximum } \\
\text { inflation } \\
\text { pressure } \\
\text { /bar }\end{array}$ \\
\hline 625 & 625 & 40 & 55 & 2.5 & 625 & 20 & 32 & 2.5 \\
\hline
\end{tabular}

\subsection{Working principle}

Due to the symmetry of the structure and the fact that each PAM can be inflated with a different air pressure the arm can achieve elongation that consequently causes bending. To be of use in different tasks, the stiffness of a PAM can be adjusted independently of its length.

When the arm reaches a certain position, through adjusting the inflating pressure of the expansive PAM and the contractile PAMs simultaneously, the stiffness can be adjusted. The principle of variable stiffness applied for the arm is shown in figure 2 in which the length of the arm remains the same. On the left hand side of Figure 2(a), the expansive PAM is inflated and the other contractile PAMs are not inflated. The status of this arm is such that it is working at lower stiffness. On the right hand side of Figure 2(a) all the four PAMs are inflated at the same time. This arm is working at higher stiffness. The working principle of the arm is reminiscent of that of a tug-of-war, shown in figure 2(b). This principle is also applied when the arm is bending. Both for straight and bent configurations, the stiffness of the arm can be changed by adjusting the inflating pressure of three contractile PAMs and that of the singular expansive PAM.

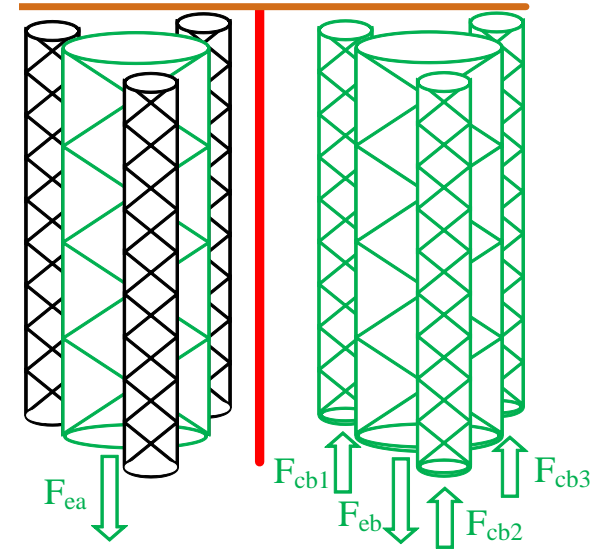

(a) Arm

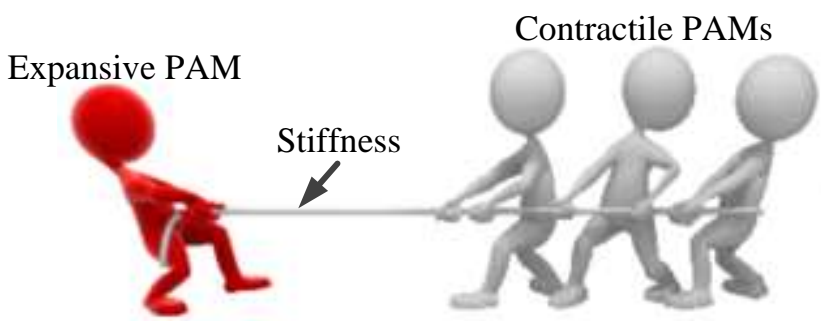

(b) Tug-of-war

Fig.2 The variable stiffness principle of the soft arm

\section{Kinematics model}

The approach to the design of kinematics models for soft robots is quite different from the approaches followed for traditional rigid robots. This is due to the fact that fixed link length and joint variables are concepts not applicable to soft robots. In order to predict the behavior of the manipulator, it was necessary to analyze the kinematics of the soft arm as well as the displacement performance of each of the three PAMs. In reference [33] and [34], the authors defined four properties which would describe the position of the free end of the PAMs relative to the base. The radius of the curve formed was defined as $\lambda$. $\phi$ was used to describe the angular displacement between the two ends of the element. $L$ was the length of the arc formed and $\theta$ was the angle the end of the element pointed relative to the base coordinate frame.

The resultant soft arm in the $o-x y z$ coordinates is shown in figure 3. It can be observed that on two ends of the arm there are two round plates. $o_{2}$ and $o_{1}$ are in the center of the top plate and the bottom plate, respectively. $A_{1}, A_{2}$ and $A_{3}$ stand for the points that the three contractile PAMs are fixed at the top plate. $B_{1}, B_{2}$ and $B_{3}$ stand for the points that the three contractile PAMs are fixed at the bottom plate. $o_{2}-x y z$ is established on the top plate and $o_{2}$ is the center of the top 
plate. $A_{1}$ is on the axis of $o_{2} x$ and $z$ is vertically upward on the top plate. Without pressure, the four PAMs are at the same length. So the length is $625 \mathrm{~mm}$.

Godage et al. ${ }^{[35]}$

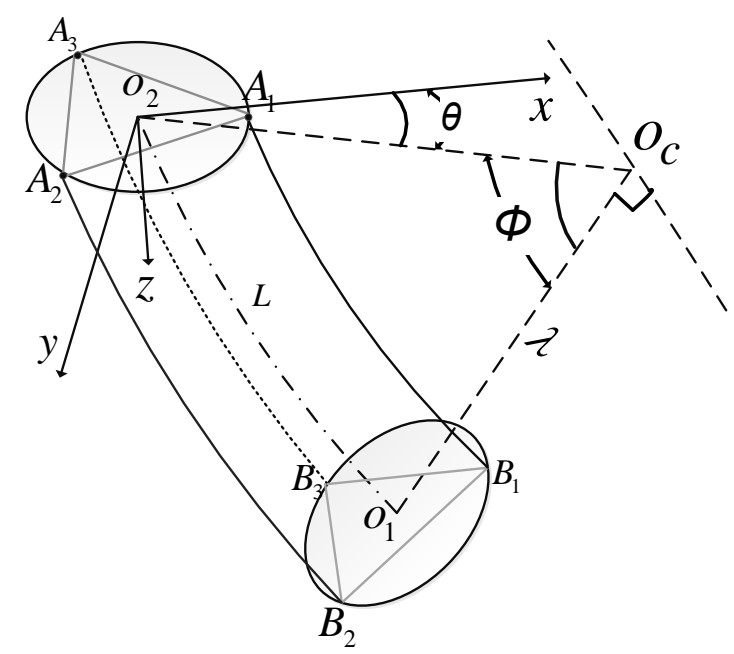

Fig.3 Kinematics of the soft arm

$$
\begin{gathered}
\lambda=\frac{\left[3\left(L_{0}+l_{0}^{\prime}\right)-l_{1}-l_{2}-l_{3}\right] r}{2 \sqrt{l_{1}^{2}+l_{2}^{2}+l_{3}^{2}-l_{1} l_{2}-l_{2} l_{3}-l_{1} l_{3}}} \\
\phi=\frac{2 \sqrt{l_{1}^{2}+l_{2}^{2}+l_{3}^{2}-l_{1} l_{2}-l_{2} l_{3}-l_{1} l_{3}}}{3 r} \\
\theta=\tan ^{-1}\left[\frac{\sqrt{3}\left(l_{3}-l_{2}\right)}{l_{2}+l_{3}-2 l_{1}}\right] \\
L_{0}+l_{0}^{\prime}+l_{0}(t)=\lambda \phi=\frac{3 L_{0}+3 l_{0}^{\prime}-l_{1}(t)-l_{2}(t)-l_{3}(t)}{3}
\end{gathered}
$$

Equations(1), (2), (3) and (4) are therefore, the kinematics model of the soft arm. The kinematics model can be used to determine the lengths $L_{1}, L_{2}$ and $L_{3}$ in the stiffness model described in the next section.

\section{Stiffness model}

\subsection{Experimental identification of the mechanical model of PAM}

According to literature review [36], a PAM can be treated like a spring and hence Hooke's law can be applied to it. The output force for the contractile PAM and the expansive PAM can thus be expressed as follows:

$$
\begin{gathered}
F_{c i}=K_{c i}\left(P_{c i}\right) \cdot\left[L_{i}-L_{c i}\left(P_{c i}\right)\right] \\
F_{e}=K_{e}\left(P_{e}\right) \cdot\left[L_{e}\left(P_{e}\right)-L\right]
\end{gathered}
$$

where, $P_{\mathrm{ci}}(\mathrm{i}=1,2,3)$ and $P_{\mathrm{e}}$ are the inflation pressures of contractile PAMs and expansive PAM respectively, (unit: bar). $K_{\mathrm{ci}}\left(P_{\mathrm{ci}}\right)(i=1,2,3)$ and $K_{\mathrm{e}}\left(P_{\mathrm{e}}\right)$ are the stiffness in $\mathrm{N} / \mathrm{m}$ for the contractile PAMs and the expansive PAM, respectively. $L_{\mathrm{ci}}\left(P_{\mathrm{ci}}\right)(i=1,2,3)$ and $L_{\mathrm{e}}\left(P_{\mathrm{e}}\right)$ are the lengths in $\mathrm{m}$ for the contractile PAMs and the expansive PAM, respectively. $K_{\mathrm{ci}}\left(P_{\mathrm{ci}}\right)$, $K_{\mathrm{e}}\left(P_{\mathrm{e}}\right), L_{\mathrm{ci}}\left(P_{\mathrm{ci}}\right)$ and $L_{\mathrm{e}}\left(P_{\mathrm{e}}\right)$ are identified through the following experiments. $L$ is the actual length of expansive PAM.

\subsubsection{Experimental set up}

An experimental test rig is shown in Figure 4. It includes a PAM, a solenoid valve, an air source triplet, an air compressor, a displacement sensor, a cylinder, a PC and a support structure. An air compressor is used as air supply to the PAM. The air source triplet is used to regulate the amount of pressure. The range of working pressure is 0-10bar. The cylinder, model SC $32 \times 75-\mathrm{S}$, is used to offer some load. The output force of cylinder can be expressed in (7). The front end of the cylinder is connected with the PAM. It is used to measure the displacement of the PAM. The air inlet of the PAM is connected with the pressure sensor to measure the pressure of the PAM. The 40PC150G2A pressure sensor has an accuracy of $\pm 2.25 \%$. In the experiment, an Arduino microcontroller is used for collecting data from the pressure sensor and displacement sensor. It also sends control signals to solenoid valve. 


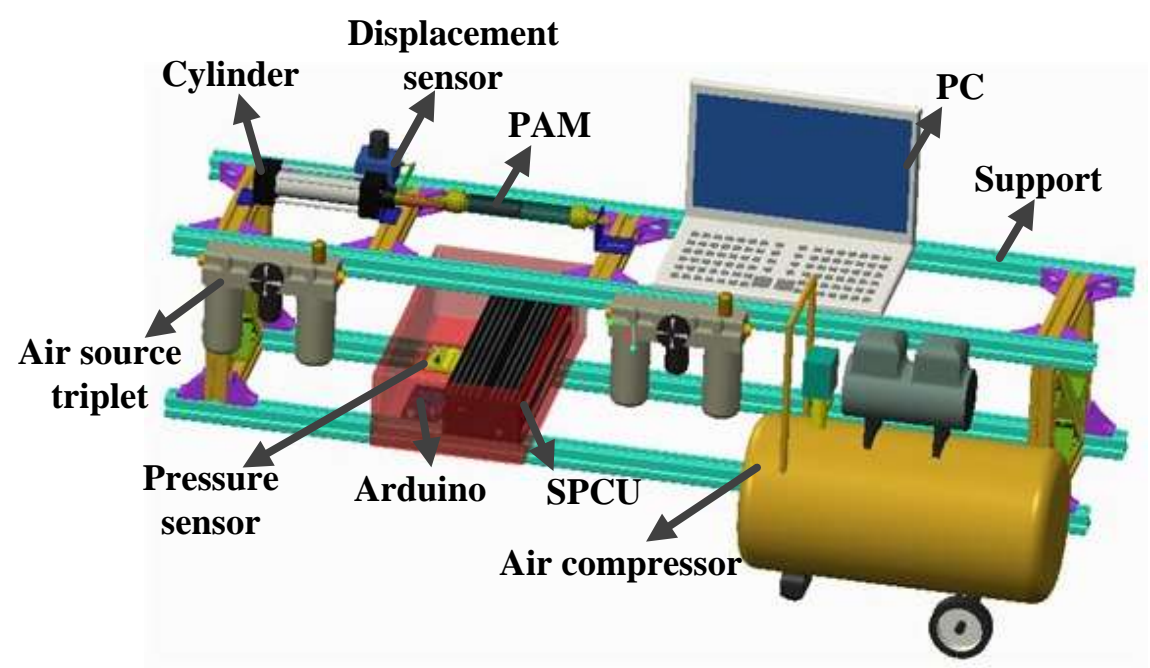

Fig.4 Schematic diagram of the experimental measurement system for pneumatic artificial muscle

$$
F_{g}=\frac{\pi}{4}\left(D^{2}-d^{2}\right) \times P
$$

where $F_{\mathrm{g}}$ is the output force of cylinder which provides load for the PAM. $D$ is the diameter of the piston, $d$ is the diameter of piston rod and $P$ is the pressure of the cylinder.

\subsubsection{Model identification}

Firstly the test rig in figure 5 is used to test the length of the PAM under no-load. The inflation pressure of cylinder is set at 0 bar, then the inflation pressures of the cylinder are set as from 0bar to $2.5 \mathrm{bar}$ in intervals of $0.2 \mathrm{bar}$. The experiment is performed under quasi-static conditions. MATLAB software is used to fit the model. The lengths of the contractile PAMs and the expansive PAM can be expressed as follows,

$$
\begin{gathered}
L_{c i}=a_{1} P_{c i}^{3}-a_{2} P_{c i}^{2}-a_{3} P_{c i}+a_{4} \quad 0<P_{c i}<2.5 \\
L_{e}=b_{1} P_{e}^{4}+b_{2} P_{e}^{3}+b_{3} P_{e}^{2}+b_{4} P_{e}+b_{5} \quad 0<P_{e}<2.5
\end{gathered}
$$

where, $a_{1}=0.0212, a_{2}=-0.0704, a_{3}=-0.00689, a_{4}=0.62844 . b_{1}=-0.03893, b_{2}=0.2421, b_{3}=-0.527, b_{4}=0.47, b_{5}=0.625$. $L_{\mathrm{ci}}\left(P_{\mathrm{ci}}\right)(i=1,2,3)$ and $L_{\mathrm{e}}\left(P_{\mathrm{e}}\right)$ are the lengths of the contractile PAMs and the expansive PAM in m, respectively.

Secondly, the test rig of Figure 5 is used to test the stiffness of the PAM. The inflation pressures of the PAMs are regulated through the air source triplet, which in this instance, is set from 0bar to $2.5 \mathrm{bar}$ at intervals of $0.5 \mathrm{bar}$. Then, the setting of the inflation pressure of cylinder is changed to between 0bar and 3bar, at an interval of $0.2 \mathrm{bar}$ so that the load is from $0 \mathrm{~N}$ to $212 \mathrm{~N}$. MATLAB software is then used again to fit the stiffness of the PAM. The stiffness of the contractile PAMs and the expansive PAM can thus be expressed as follows,

$$
\begin{gathered}
K_{c i}=106 \times P_{c i}^{3}-596 \times P_{c i}^{2}+1275 P_{c i}+275.64 \\
K_{e}=-18.9 \times P_{e}^{3}+1.9048 \times P_{e}^{2}+374.48 P_{e}+139.4
\end{gathered}
$$

where, $K_{\mathrm{ci}}\left(P_{\mathrm{ci}}\right)(i=1,2,3)$ and $K_{\mathrm{e}}\left(P_{\mathrm{e}}\right)$ are the stiffness of the three contractile PAMs and the expansive PAM in $\mathrm{m}$, respectively. According to the equations of (5) and (6), PAMs still possesses some stiffness under no inflation pressure. This stiffness characteristics largely depends on the material characteristics of elastic tube and external woven braided shell.

\subsection{Stiffness model of the arm}

While the soft arm is in the fixed bent position shown in Figure 5, the arm is under force balance. The forces generated by both contractor and extensor PAMs are perpendicular to the bottom mounting plate. Force analysis is conducted on the arm, and the force analysis is in Figure 6, because the output force direction of PAM muscles are inverse, the cross symbol is used to express the output force of contractile PAMs, and the dot symbol is used to express the output force of expansive PAM. In order to determine the axis stiffness, there is force a $F$ applied on the soft arm, which causes the changing of the length, according to force balance and moment balance, then the forces can be expressed as follows,

$$
\begin{gathered}
F_{c 1}+F_{c 2}+F_{c 3}=F_{e}+F \\
F_{c 1} \cdot r+F_{c 2} \cdot r=F_{c 3} \cdot 2 r \\
F_{c 1} \cdot l_{1}=F_{c 2} \cdot l_{2}
\end{gathered}
$$

where $F_{\mathrm{c} 1}, F_{\mathrm{c} 2}$ and $F_{\mathrm{c} 3}$ are output force for the three contractile PAMs respectively. $r$ is the radius of three PAMs, and the 
distances from $F_{\mathrm{c} 1}, F_{\mathrm{c} 2}$ to the axis of $y 1$ are $l_{1}, l_{2}$ respectively. Then the equations can be simplified as follows,

$$
F_{c 1}=F_{c 2}=F_{c 3}=\frac{F_{e}+F}{3}
$$

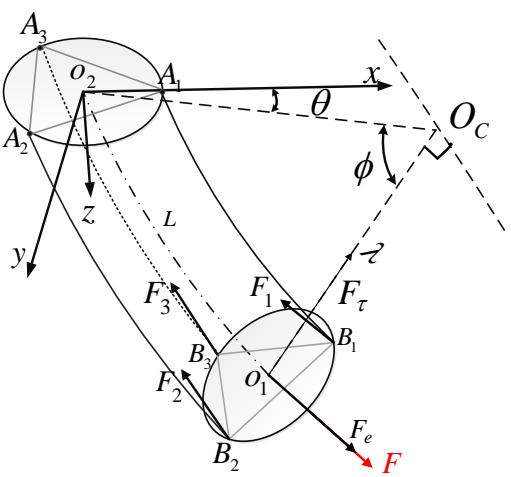

(a) Visualisation of orientation of forces of the arm

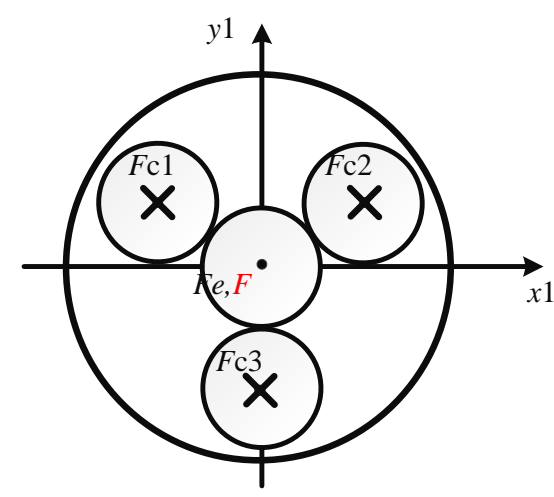

(b) Location of point of force application on the bottom plate c and force analysis

If (5) and (6) are substituted it into (15) respectively, then it can be expressed as follows,

$$
\begin{aligned}
& L_{1}=\frac{K_{e}\left(P_{e}\right) \cdot\left[L_{e}\left(P_{e}\right)-L\right]+F}{3 K_{c 1}}+L_{c 1}\left(P_{c 1}\right) \\
& L_{2}=\frac{K_{e}\left(P_{e}\right) \cdot\left[L_{e}\left(P_{e}\right)-L\right]+F}{3 K_{c 2}}+L_{c 2}\left(P_{c 2}\right) \\
& L_{3}=\frac{K_{e}\left(P_{e}\right) \cdot\left[L_{e}\left(P_{e}\right)-L\right]+F}{3 K_{c 3}}+L_{c 3}\left(P_{c 3}\right)
\end{aligned}
$$

If (16), (17) and (18) are substituted into (15), then $L_{1}, L_{2}$ and $L_{3}$ can be eliminated. So, it can be expressed as follows,

$$
F=\frac{9 L A+K_{e} L B-K_{e} L_{e}-3 A\left(L_{c 1}+L_{c 2}+L_{c 3}\right)}{B}
$$

where,

$$
A=K_{c 1} K_{c 2} K_{c 3} \quad B=\left(K_{c 1} K_{c 2}+K_{c 1} K_{c 3}+K_{c 3} K_{c 2}\right)
$$

Under different inflation pressure, the stiffness of the arm can be expressed as follows,

$$
K_{\text {Arm }}=\frac{d F}{d L}
$$

If a derivative of (20) is taken, then stiffness of arm can be shown as follows,

$$
K_{\text {Arm }}=\frac{9 K_{c 1} K_{c 2} K_{c 3}+K_{e}\left(K_{c 1} K_{c 2}+K_{c 2} K_{c 3}+K_{c 1} K_{c 3}\right)}{K_{c 1} K_{c 2}+K_{c 2} K_{c 3}+K_{c 1} K_{c 3}}
$$

\section{Experimental Verification of stiffness model}

\subsection{Test rig}

In order to verify the accuracy of the stiffness model of the soft arm, a schematic diagram of the stiffness tests and the figure of the test rig are shown in Figure 6 and Figure 7 respectively.

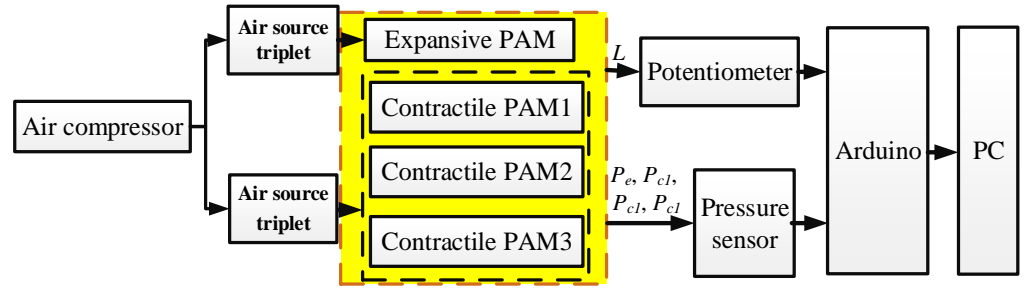

Fig.6 Schematic diagram of stiffness tests. 


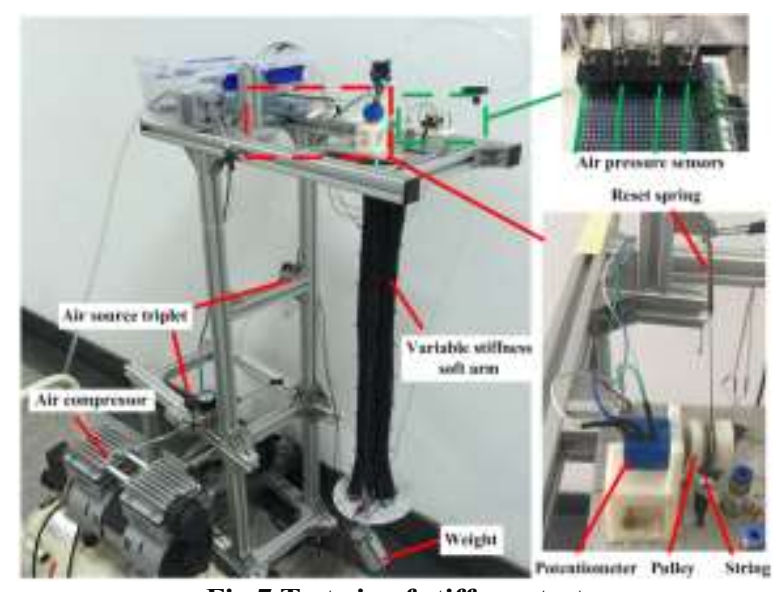

Fig.7 Test rig of stiffness test

Figure 7 shows the test rig, which includes an air compressor, air source triplet, $1 \mathrm{~kg}$ weight, four air pressure sensors, reset spring, potentiometer, pulley, and string. In the experiment, the air compressor is used for air supply for the PAM. The air source triplet is used to regulate the inflation pressure of the PAM. The pressure sensors are used to read the inflation pressure of the PAM. One end of the string is connected with the reset spring. The other end is connected with the bottom plate of soft arm and is further connected on the expansive PAM by nylon ties. When the arm starts to move, the length of the soft arm will change. Then pulley will start to turn and the resistance will also change. The changes in resistance can be calculated by measuring the length of the soft arm. The Arduino microcontroller will collect the data from the potentiometer in real time. The weight is used to provide the load for the arm.

\subsection{Experimental Test}

In the experiment, before loading, the inflation pressure of the four PAMs is selected so the arm keeps the length of $610 \mathrm{~mm}$. Firstly, the expansive PAM is inflated and the arm will elongate as a result. Then the inflation pressure of the three contractile PAMs is regulated and the length of the arm is decreased back to $610 \mathrm{~mm}$. The expansive PAM is set from 0bar to 2.50bar with intervals of 0.50bar. The three contractile PAMs are set at $0.80 \mathrm{bar}, 0.90 \mathrm{bar}, 1 \mathrm{bar}, 1.20 \mathrm{bar}$, $1.40 \mathrm{bar}$ and $1.45 \mathrm{bar}$, respectively. Secondly, $1 \mathrm{~kg}$ weight is loaded on the bottom end of the soft arm and the length variation is collected through the Arduino. These experiments are repeated three times and the mean values are calculated. The test results are shown in figure 8 .

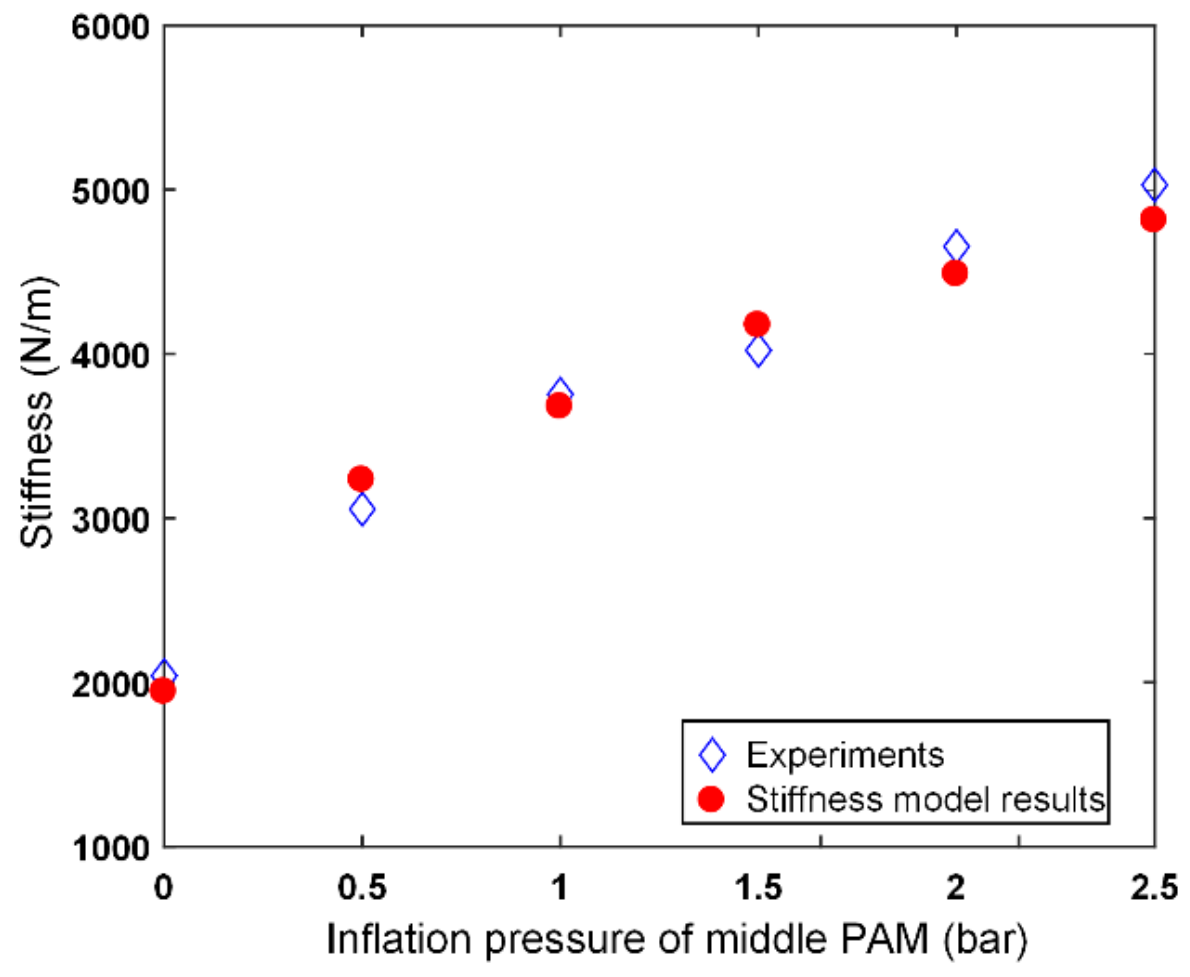

Fig. 8 Comparison schematics of experiment value and theoretical value 
From figure 8, it can be observed that the experimental results and the results of the theoretical stiffness model both possess the same trend. The mean error is $4.09 \%$ and the maximum error is $5.56 \%$.

In order to test the stiffness of the arm in a bending position, the pressure of two contractile PAMs are set at equal pressure, while the pressure of the third PAM is set differently, the arm will be bent. The length of the arm is also adjusted at $610 \mathrm{~mm}$. Firstly, the expansive PAM is inflated and the arm will elongate as a result. Then the inflation pressure of the three contractile PAMs is regulated (the pressure of the two of the PAMs are adjusted equally, and the pressure of the third PAM is adjusted differently from the other two.) and the length of the arm is decreased back to $610 \mathrm{~mm}$. The expansive PAM is set from 0 bar to $2.50 \mathrm{bar}$ with intervals of $0.50 \mathrm{bar}$. The two contractile PAMs are set at $0.60 \mathrm{bar}, 0.80 \mathrm{bar}$, 0.85bar, 0.90bar, 1.1 bar and 1.2bar, respectively. The third PAM is set at $0.90 \mathrm{bar}, 1.20 \mathrm{bar}, 1.30 \mathrm{bar}, 1.33 \mathrm{bar}, 1.60 \mathrm{bar}$ and $1.65 \mathrm{bar}$, respectively. A dynamometer is applied to the center of the bottom plate, then the length variation and the data from the dynamometer are collected. Then the stiffness of the arm are calculated, and the results are shown in figure 9.

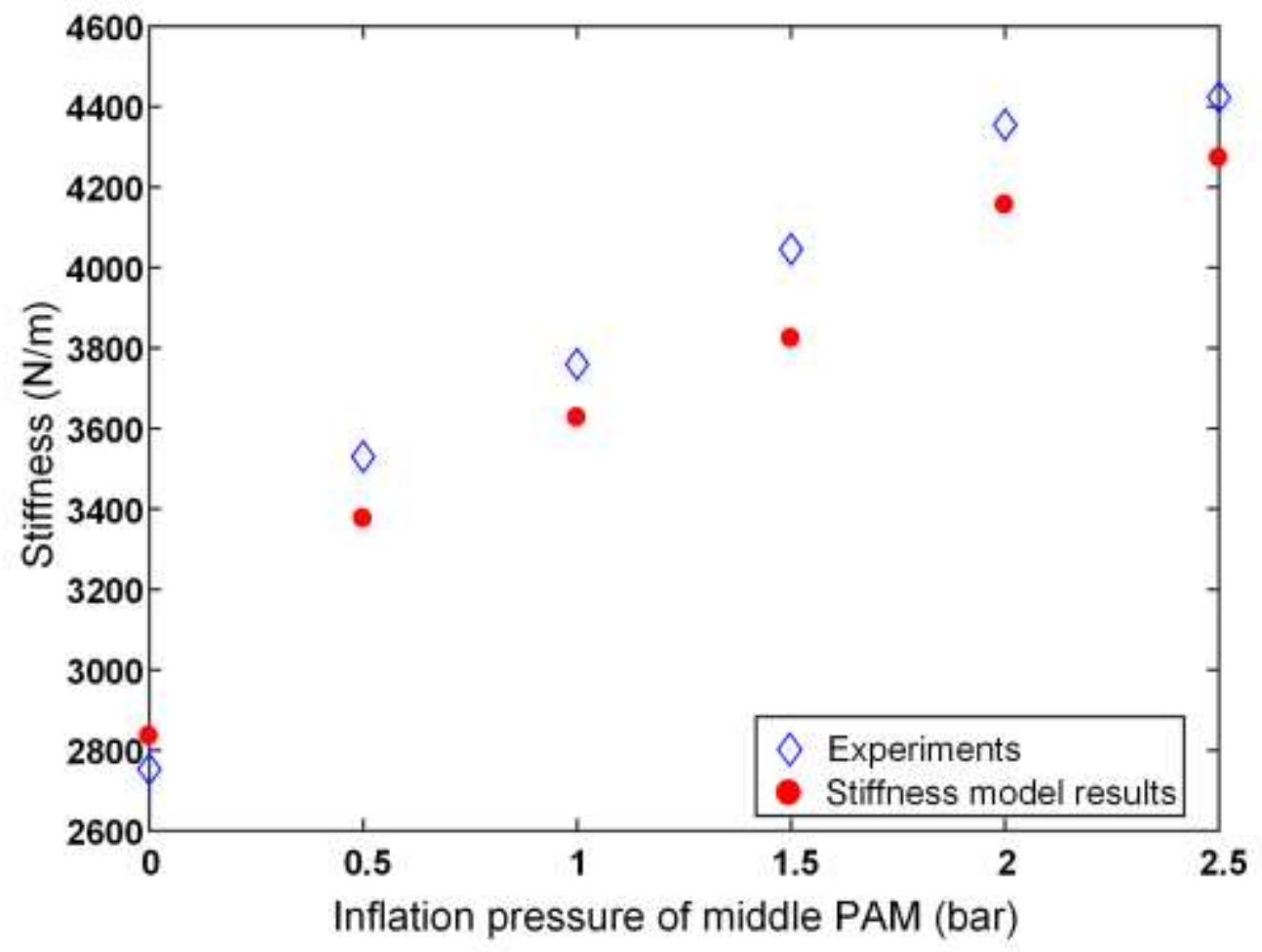

Fig. 9 Comparison schematics of experiment value and theoretical value in a bending position

From figure 9, it can be observed that in the stiffness test of the arm in a bending position the experimental results and the results of the theoretical stiffness model also both possess the same trend. The mean error is $4.30 \%$ and the maximum error is $6.33 \%$. The reason for the error is that the theoretical stiffness model does not take friction and the influence of the ties into account.

\section{Stiffness and position control}

\subsection{Auto/Active Disturbances Rejection Controller (ADRC)}

An ADRC controller includes a Tracking Differentiator (TD), an Extended State Observer (ESO) and a Nonlinear State Error Feedback (NLSEF). The structure diagram of a second order ADRC system is shown in figure 10.

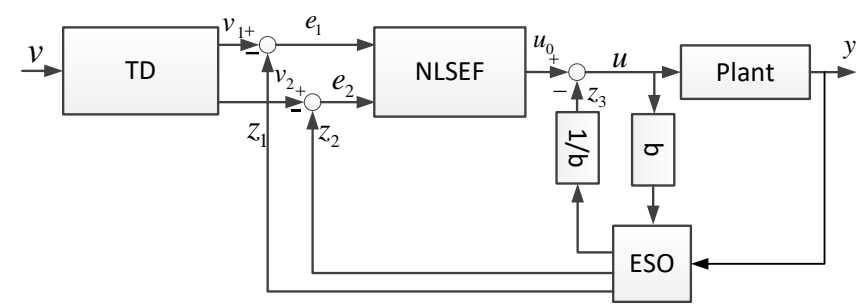

Fig. 10 The structure diagram of a second order ADRC system

Taking the second order system (22) as an example, the second order discrete ADRC specific algorithm will be as 
shown in formula (23),

$$
\begin{aligned}
& \left\{\begin{array}{l}
\&=x_{2} \\
\&=u
\end{array}\right. \\
& \text { TD: }\left\{\begin{array}{l}
v_{1}(t+1)=v_{1}(t)+T * v_{2}(t) \\
v_{2}(t+1)=v_{2}(t)+T * \text { fhan }\left(v_{1}(t)-v(t), v_{2}, r_{0}, h\right)
\end{array}\right. \\
& \text { ESO }\left\{\begin{array}{l}
e=z_{1}(t)-y(t) \\
f e=f a l(e, 0.5, h) \\
f e_{1}=f a l(e, 0.25, h) \\
z_{1}(t+1)=z_{1}(t)+h *\left(z_{2}(t)-\beta_{01} e\right) \\
z_{2}(t+1)=z_{2}(t)+h *\left(z_{3}(t)-\beta_{02} f e+u\right) \\
z_{3}(t+1)=z_{3}(t)+h^{*}\left(-\beta_{03} f e_{1}\right)
\end{array}\right. \\
& \mathrm{NSEF}:\left\{\begin{array}{l}
e_{1}=v_{1}(t)-z_{1}(t) \\
e_{2}=v_{2}(t)-z_{2}(t) \\
u_{0}=\beta_{1} \operatorname{fhan}\left(e_{1}, r, h_{1}\right)+\beta_{2} \operatorname{fhan}\left(e_{2}, r, h_{1}\right)
\end{array}\right. \\
& \mathrm{u}: \mathrm{u}(t)=u_{0}-z_{3}(t)
\end{aligned}
$$

where,

$$
f a l(e, a, d)=\left\{\begin{array}{l}
e^{*} d^{(1-a)},|e|<d \\
|e|^{a} * \operatorname{sign}(e),|e|>=d
\end{array}\right.
$$

The computational method of $\operatorname{fhan}\left(x_{1}, x_{2}, r, h\right)$ is shown as follows,

$$
\begin{aligned}
& \begin{array}{l}
d=r h \\
d_{0}=h d \\
y=x_{1}+h x_{2}
\end{array} \\
& a_{0}= \begin{cases}x_{2}+\frac{\left(a_{0}-d\right)}{2} \operatorname{sign}(y), \quad|y|>d_{0} \\
x_{2}+\frac{y}{h}, \quad|y|<=d_{0}\end{cases} \\
& \text { fhan }=-\left\{\begin{array}{l}
r \operatorname{sign}(a),|a|>d \\
r \frac{a}{d},|a|<=d
\end{array}\right.
\end{aligned}
$$

TD: $v(t)$ is the input signal, $v_{1}(t)$ is the tracking signal of $v(t)$, T is the sample time, $a$ is non-linear factor, $h$ is filter factor and $R$ is velocity factor.

ESO: $y(t)$ is the output of the system, $z_{1}(t)$ is the tracking signal of $y(t), z_{2}(t)$ is the differential signaling of $z_{1}(t), z_{3}(t)$ is the disturbance signal of the system, $e$ is the error signal, $\beta_{01}, \beta_{02}$, and $\beta_{03}$ is the gain of error correction, and the algorithm of $\operatorname{fal}(\cdot)$ is in (24).

NLSEF: $e_{1}$ and $e_{2}$ are error and differential signal respectively, $\beta_{1}$ and $\beta_{2}$ are error gain and differential gain.

\subsection{FADRC}

Three main parts of an ADRC are required and these are designed separately. This also allows the parameter settings for the ADRC to be adjusted separately. $\mathrm{h}$ and $\mathrm{R}$ of TD are fixed parameters which do not need to be adjusted manually. However, $\beta_{01}, \beta_{02}$ and $\beta_{03}$ of ESO and $\beta_{1}$ and $\beta_{2}$ of NLSEF need to be adjusted manually and this brings much more inconvenience in real applications. In this paper, a Fuzzy adaptive algorithm has been combined with the ADRC. The parameters of ADRC are then adjusted through the Fuzzy adaptive algorithm. In general, for input variables, the fuzzy controller selects the given values, the deviation of the output value and the deviation change rate. It implements the control law or the increment of its control and presents it as the output. The basic principle of the fuzzy controller is shown in the block diagram of figure 11. 


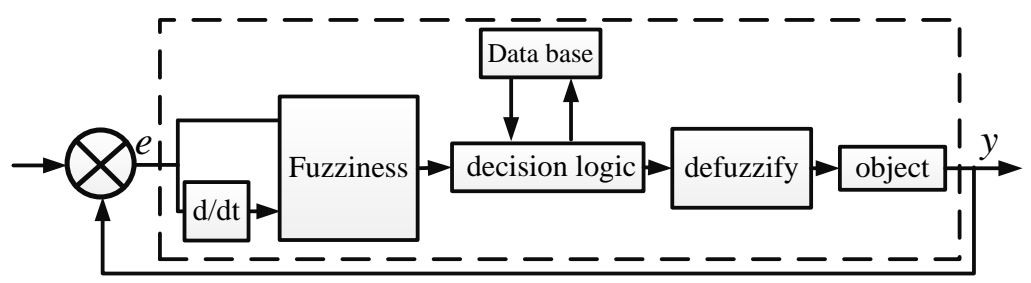

Fig.11 Block Diagram of Fuzzy Control System

From figure 11, the ESO fuzzy variables are $e, e^{\prime}, \Delta \beta_{01}, \Delta \beta_{02}$ and $\Delta \beta_{03}$. The ESO NLSEF fuzzy variables are $e_{1}, e_{2}$, $\Delta \beta_{1}$ and $\Delta \beta_{2}$. The membership function of $e, e^{\prime}, e_{1}$ and $\mathrm{e}_{2}$ is the gaumssf. The membership function of $\Delta \beta_{01}, \Delta \beta_{02}, \Delta \beta_{03}$, $\Delta \beta_{1}$ and $\Delta \beta_{2}$ is trimf. The Mamdani method is selected for fuzzy deduction in which the method of defuzzifying is the weighted average. For $\left(\Delta \beta_{01}, \Delta \beta_{02}, \Delta \beta_{03}, \Delta \beta_{1}, \Delta \beta_{2}\right)$, the fuzzy rule is given in table 2 .

Tab.2 $\Delta \beta_{01}, \Delta \beta_{02}, \Delta \beta_{03}, \Delta \beta_{1}$ and $\Delta \beta_{2}$ fuzzy rule table

\begin{tabular}{cccccccc}
\hline$e^{\prime} / e_{1}$ & \multicolumn{7}{c}{$e / e_{2}$} \\
\cline { 2 - 7 } NB & NB & NM & NS & ZO & PS & PM & PB \\
NM & PB & PB & PM & PM & PM & ZO & ZO \\
NS & PM & PM & PM & PS & PS & NS & NS \\
ZO & PM & PM & PS & ZO & NS & NM & NM \\
PS & PS & PS & ZO & NS & NS & NM & NM \\
PM & PS & ZO & NS & NM & NM & NM & NB \\
PB & ZO & ZO & NM & NM & NM & NB & NB \\
\hline
\end{tabular}

According to the fuzzy rule table, the membership function and fuzzy deduction, $\Delta \beta_{01}, \Delta \beta_{02}, \Delta \beta_{03}, \Delta \beta_{1}$ and $\Delta \beta_{2}$ can be checked out. The they can be substituted into the following function:

$$
\left\{\begin{array}{l}
\beta_{01}=\beta_{01}^{\prime}+\Delta \beta_{01} \\
\beta_{02}=\beta_{02}^{\prime}+\Delta \beta_{02} \\
\beta_{03}=\beta_{03}^{\prime}+\Delta \beta_{03} \\
\beta_{1}=\beta_{1}^{\prime}+\Delta \beta_{1} \\
\beta_{2}=\beta_{2}^{\prime}+\Delta \beta_{2}
\end{array}\right.
$$

where, $\beta_{01}^{\prime}, \beta_{02}^{\prime}, \beta_{03}^{\prime}, \beta_{1}^{\prime}$ and $\beta_{2}^{\prime}$ are the initial values. According to equation (21), $\beta_{01}, \beta_{02}, \beta_{03}, \beta_{1}$, and $\beta_{2}$, can also be calculated. Then, FADRC can be designed. Based on the reference ${ }^{[28]}$, the schematic of FADRC is shown in figure 12 .

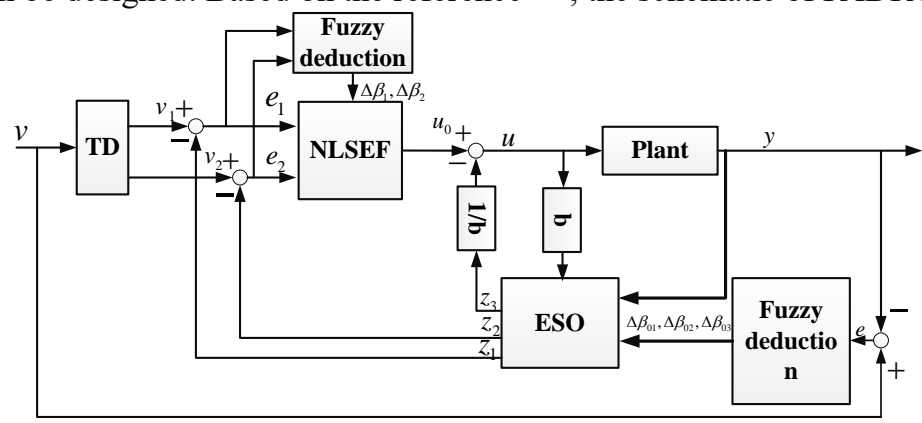

Fig.12 Schematic of FADRC

\section{Experimental verification of FADRC control}

\subsection{Control system design of Soft arm of FADRC control}

A soft arm driven by pneumatic muscles possesses strong nonlinearity. In this paper, FADRC is used to control the stiffness and position of the design arm. Based on the kinematics model and the stiffness model, the lengths of the three contractile PAMs are controlled and pressure of the expansive PAM is also controlled. The control schematic diagram of the system required to control the position and stiffness is shown in figure 13. 


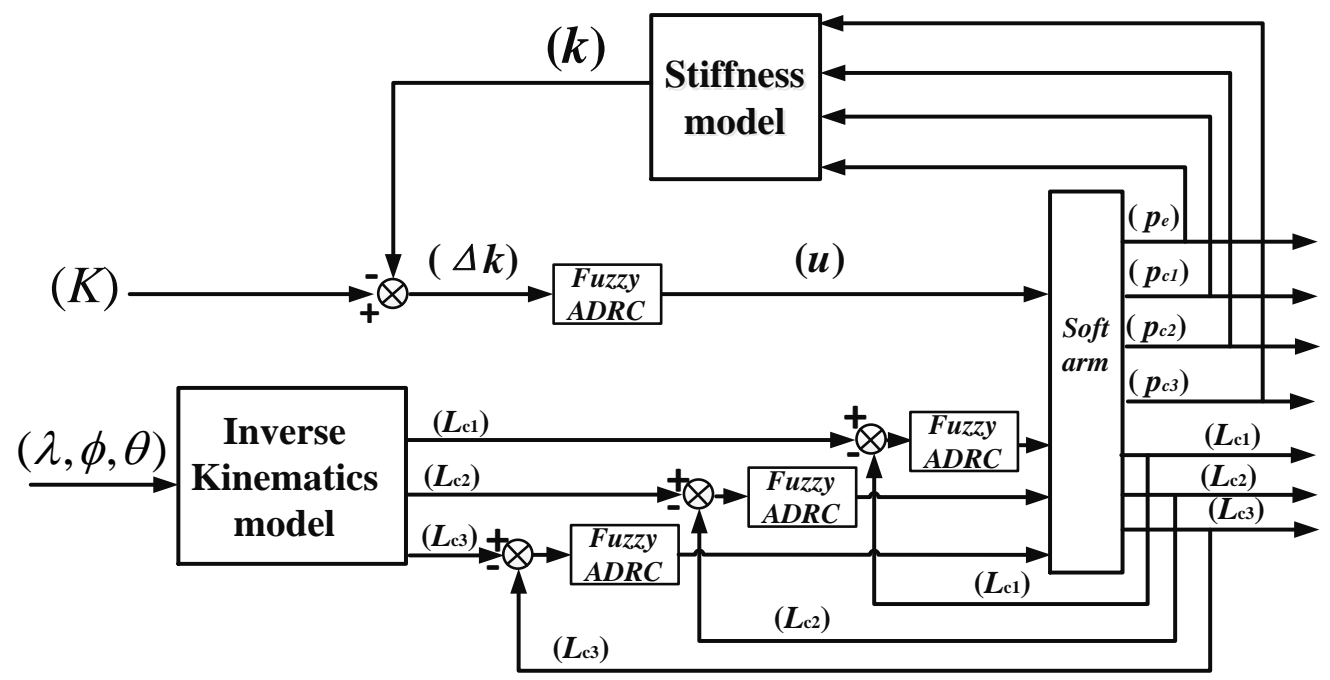

Fig.13 Control schematic diagram of position and stiffness control system

\subsection{Experimental platform setup}

The test rig of figure 7 is changed to the rig shown in figure 14. The three potentiometers shown in Figure 14 are used to measure the length of the three contractile PAMs. The four PAMs are controlled through a MATRIX solenoid valve. The Arduino is used to collect the data from the pressure sensors and the potentiometers and then send the data to MATLAB.

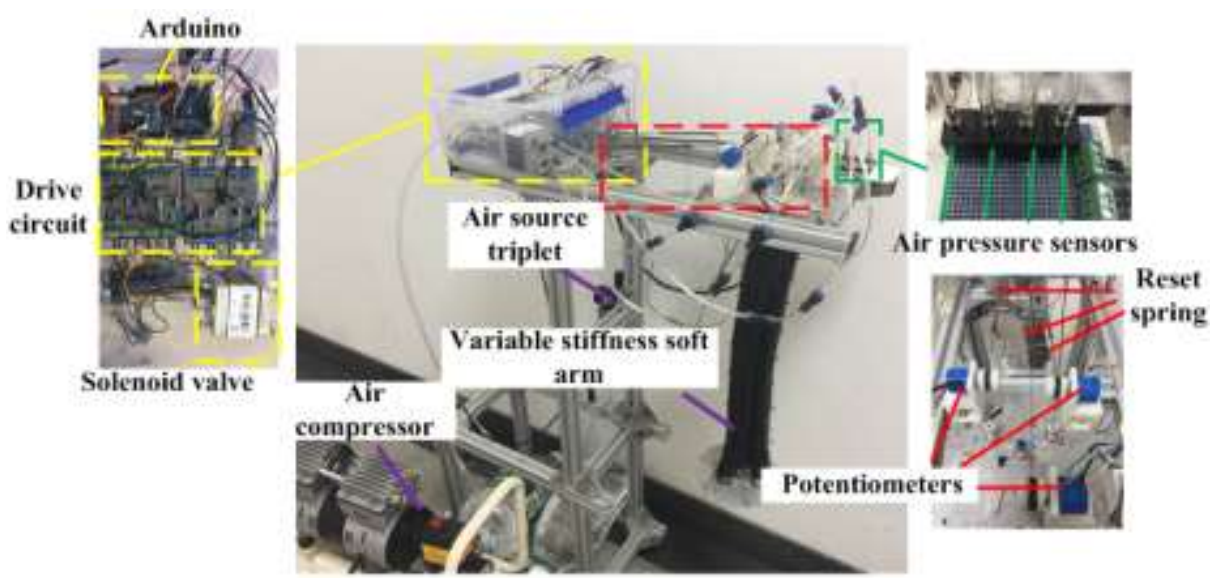

Fig.14 Test rig of control system

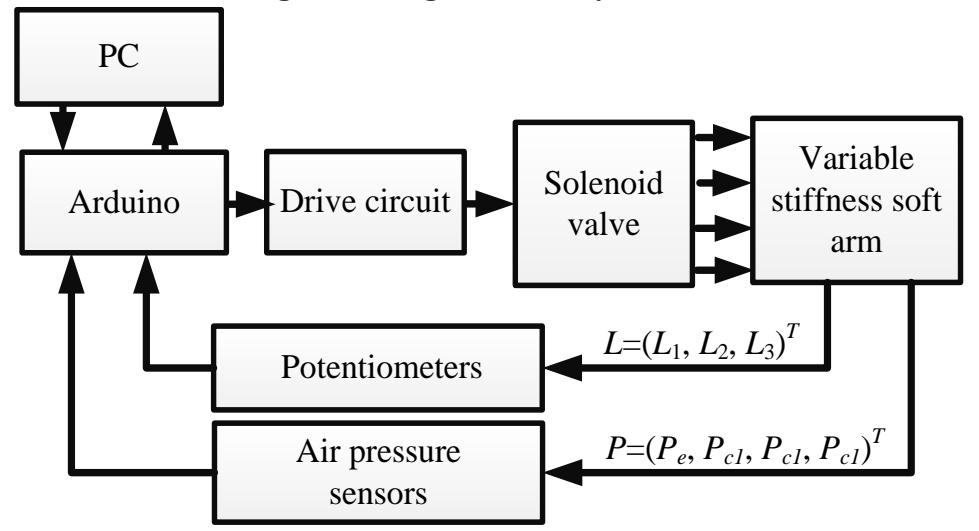

Fig.15 The principle diagram of control test

\subsection{Experiments}

To investigate the control effect of the FADRC controller, a PID controller and FADRC controller were applied to control the soft arm. Using the test rig of Figure 14, experiments on the soft arm were carried out under no-load and $500 \mathrm{~g}$ load, respectively.

\subsubsection{Stiffness Control Experiments}

Firstly stiffness control experiments were carried out. The arm is controlled to track a step signal, and stiffness of 
the arm is controlled to track a square signal. The goal of this experiments is to test the stiffness controllability of this arm. A step signal with an amplitude of 590mm, $610 \mathrm{~mm}$ and $610 \mathrm{~mm}$ was applied as reference signal for the three PAMs, respectively. The three contractile PAMs have different lengths, thus the arm will be bent. The pressure of the expansive PAM is controlled as an intermediate variable of the stiffness of the arm. The reference signal for stiffness is a square signal of $3000 \mathrm{~N} / \mathrm{m}-3800 \mathrm{~N} / \mathrm{m}$ in amplitude, with a period of $30 \mathrm{~s}$. The control parameters of the FADRC controller for the contractile PAMs are $r=2000, h=0.001, T=0.001, \beta_{01}=1, \beta_{02}=0.33, \beta_{03}=1 / 27, \beta_{1}=90, \beta_{2}=150, a_{1}=0.8, b=1.5, d=0.01$. The control parameters of FADRC for the expansive PAMs are $r=1000, h=0.001, T=0.001, \beta_{01}=1, \beta_{02}=0.33, \beta_{03}=1 / 27, \beta_{1}=0.9$, $\beta_{2}=0.00003, a_{1}=0.4, a_{2}=1.1, b=1.4, d=0.01$.

The control parameters of PID for the three contractile PAMs are $\mathrm{P}=4, \mathrm{I}=0.3$, and $\mathrm{D}=0$. The control parameters of PID for the expansive PAM are $\mathrm{P}=0.16, \mathrm{I}=0$, and $\mathrm{D}=0$. The control results of position and stiffness for the soft arm under no load and $500 \mathrm{~g}$ load are shown in figure 16 and figure 17.

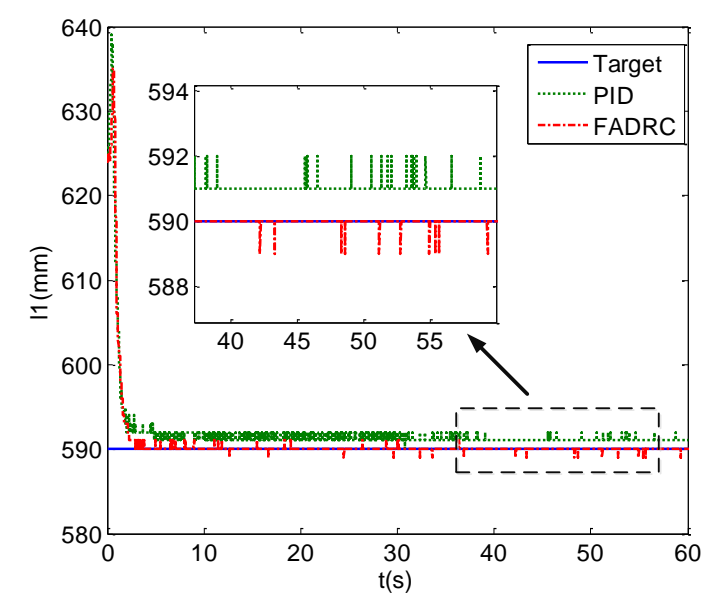

(a1)

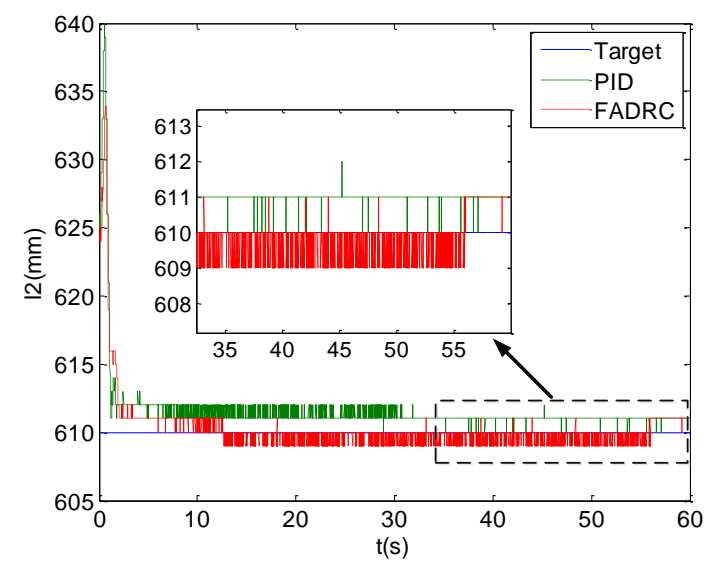

(b1)

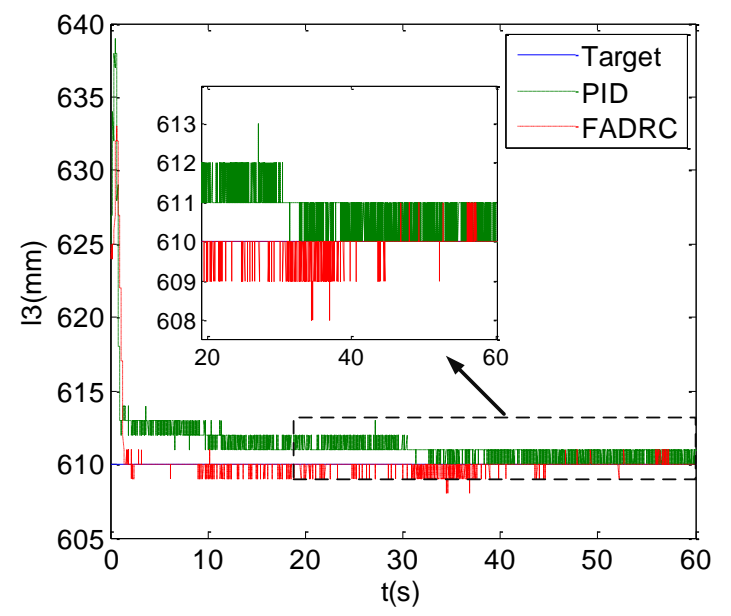

(c1)

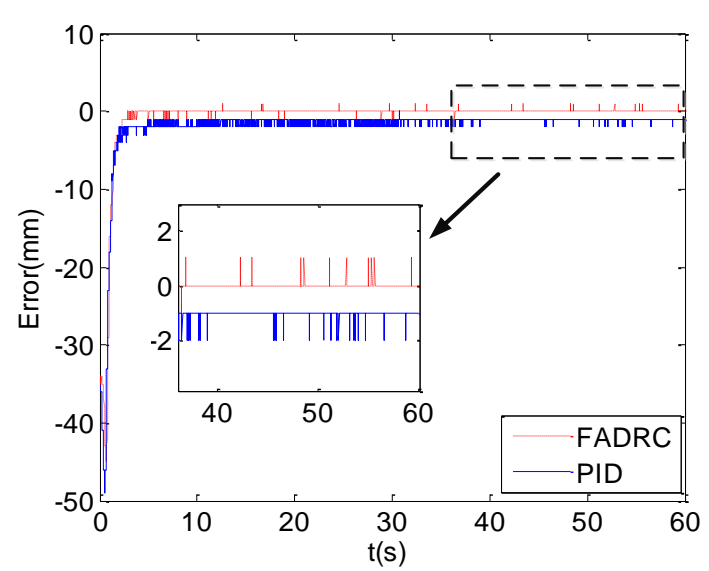

(a2)

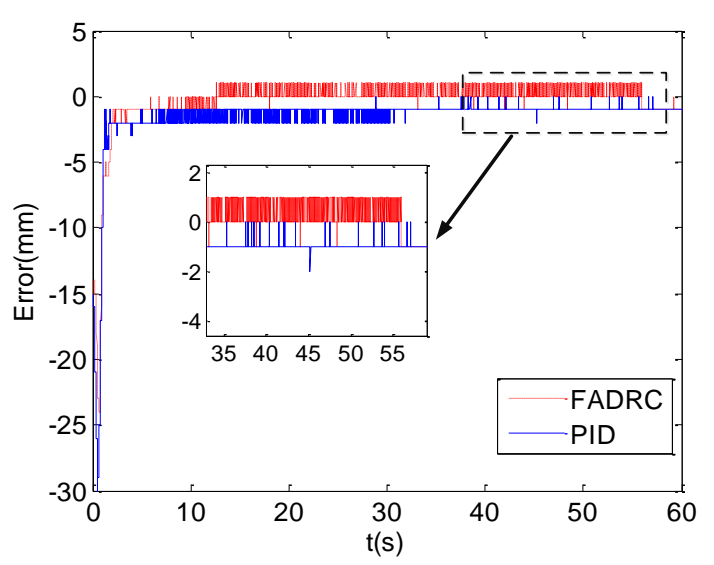

(b2)

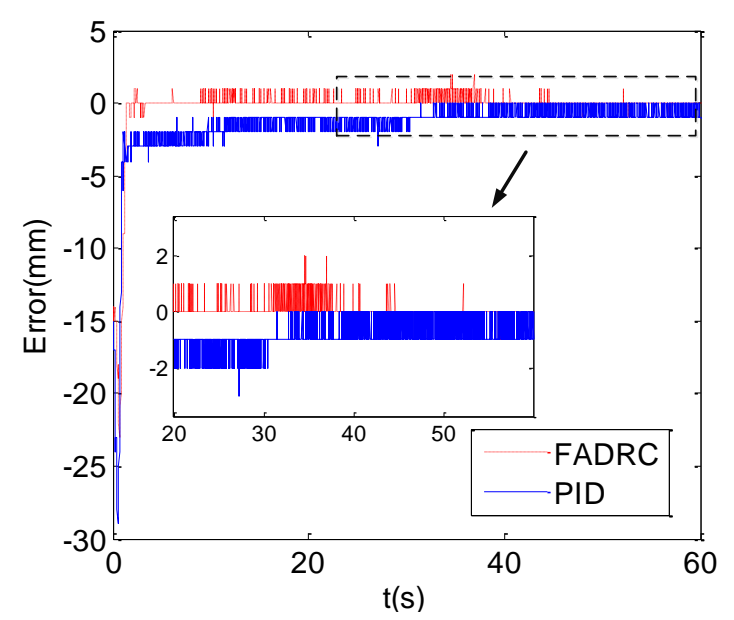

(c2) 


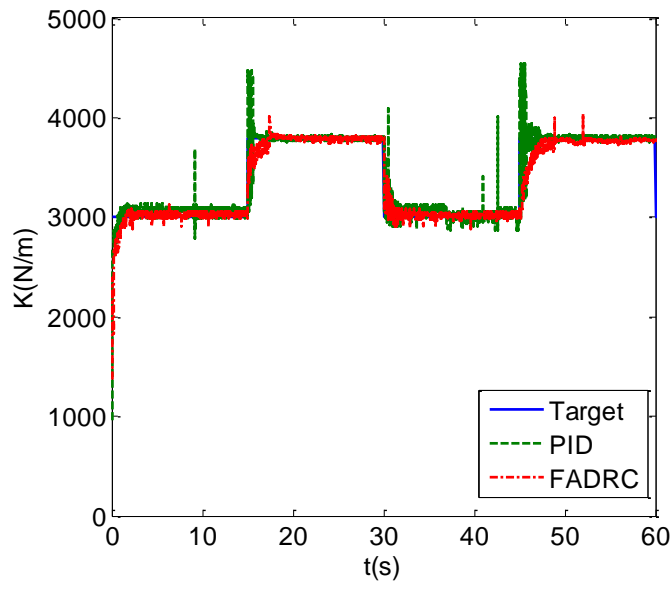

(d1)

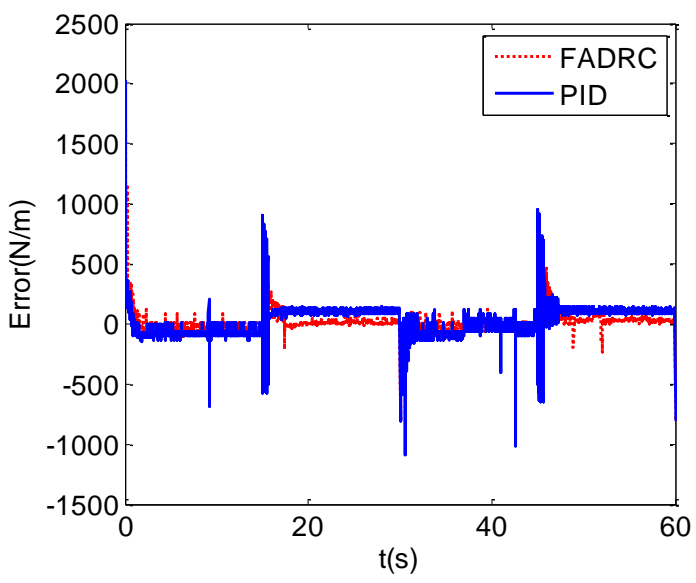

(d2)

Fig.16 Under no load, experiment results of stiffness and position control of soft arm. (a1), (b1) and (c1) stand for the length of three contractile PAMs respectively, and (d1) stands for the stiffness of the soft arm. (a2), (b2), (c2) and (d2) are the corresponding error of (a1), (b1), (c1) and (d1).

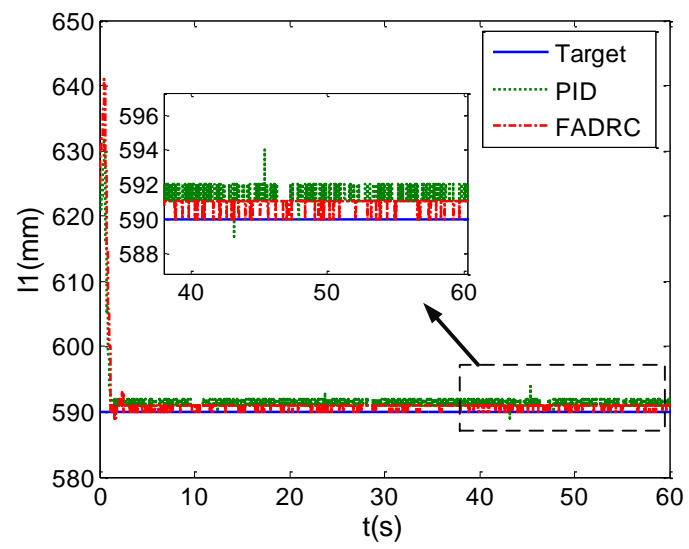

(a1)

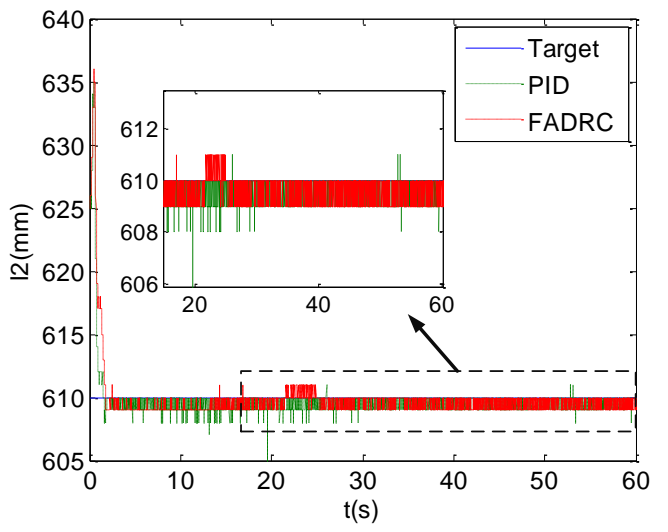

(b1)

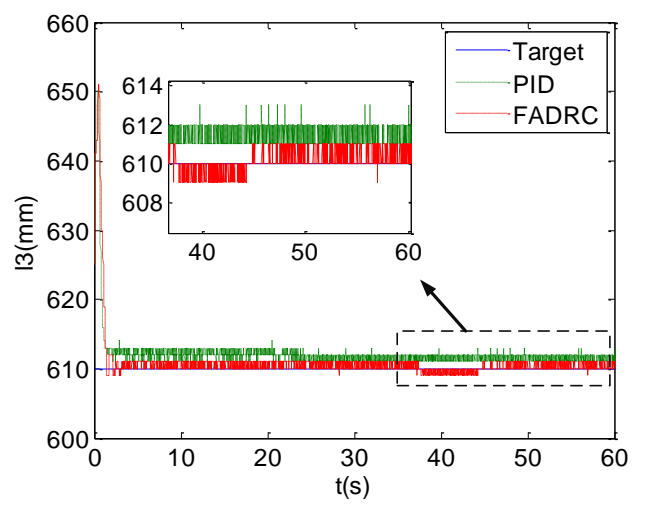

(c1)

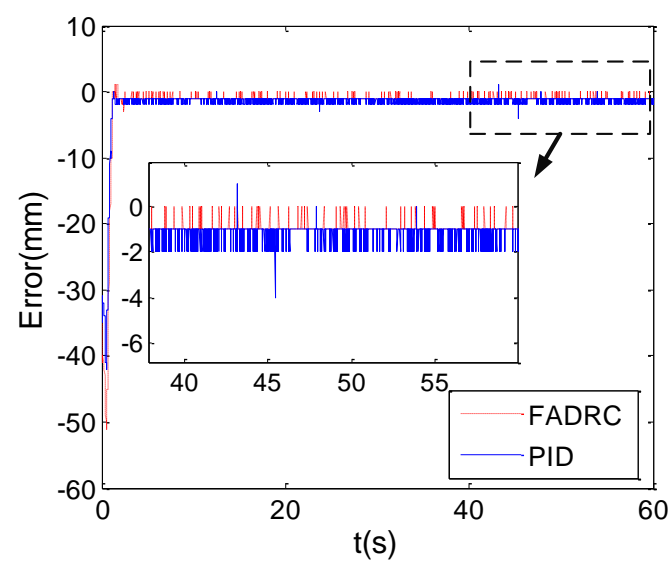

(a2)

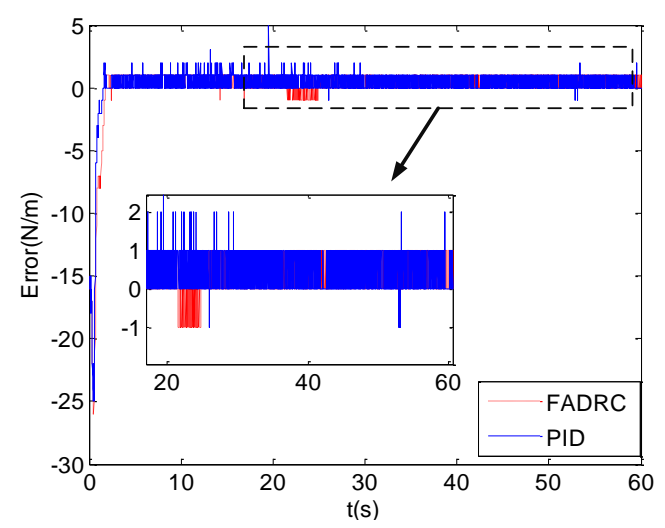

(b2)

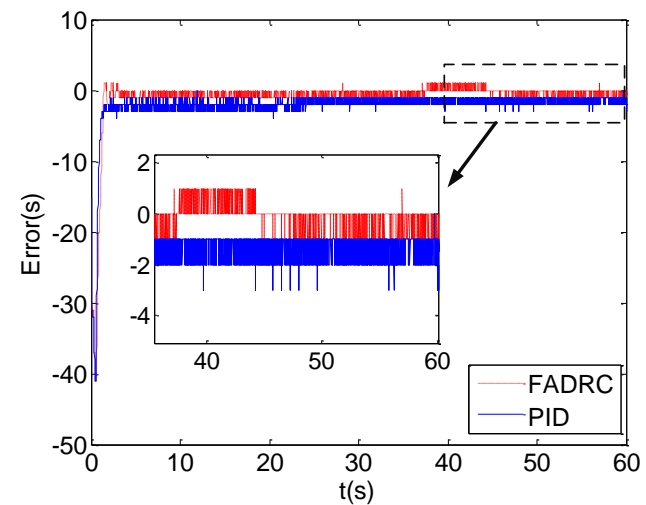

(c2) 


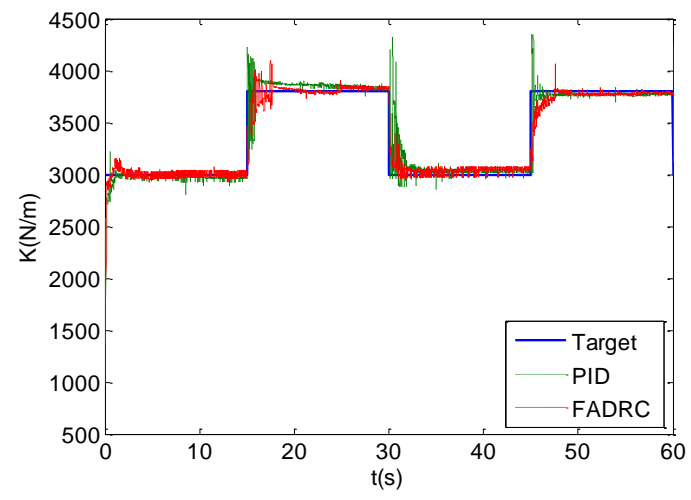

(d1)

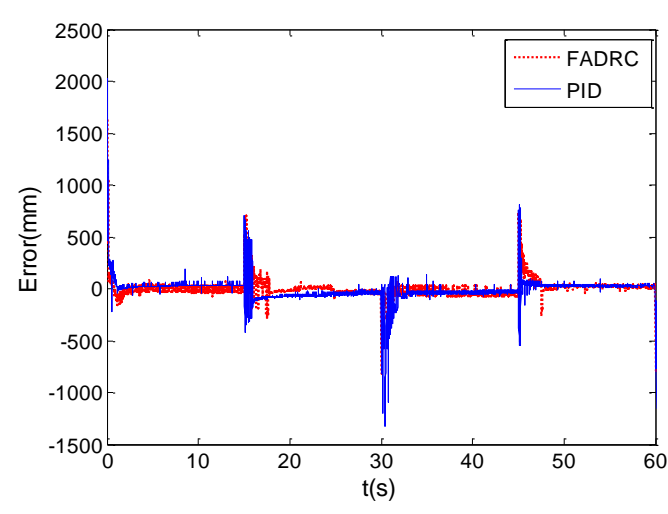

$(\mathrm{d} 2)$

Fig. 17 Under $500 \mathrm{~g}$ load, experiment results of stiffness and position control of soft arm. (a1), (b1) and (c1) stand for the length of three contractile PAMs respectively, and (d1) stands for the stiffness of the soft arm. (a2), (b2), (c2) and (d2) are the corresponding error of (a1), (b1), (c1) and (d1).

Figure 16 and 17 are the control results of PID and FADRC respectively. $L_{1}, L_{2}$ and $L_{3}$ stand for the length of three contractile PAMs. These two controllers can achieve the control of position and variable stiffness of the arm under no load or $500 \mathrm{~g}$ load. The FADRC is obviously better than the traditional PID controller, because FADRC possesses a relatively lower overshoot and better accuracy. Based on figure $16(\mathrm{~d} 1)$, the average error of PID and FADRC is $105.27 \mathrm{~N} / \mathrm{m}$ and $55.37 \mathrm{~N} / \mathrm{m}$, respectively. Based on Figure $17(\mathrm{~d} 1)$, the average error rate of PID and FADRC is $58.22 \mathrm{~N} / \mathrm{m}$ and $51.31 \mathrm{~N} / \mathrm{m} \%$, respectively. From the position control results of the soft arm of figure 16 and 17, FADRC is also better than the PID. The position control of maximum steady-state average error rate of PID and FADRC after $20 \mathrm{~s}$ in figure 16 is $0.42 \%$ and $0.18 \%$, respectively. Under $500 \mathrm{~g}$, the position control results of PID and FADRC in figure 17 is $0.21 \%$ and $0.17 \%$, respectively. Under $500 \mathrm{~g}$, FADRC also possesses good control results, which means this controller has good adaptability.

\subsubsection{Position Control Experiments}

In this section, the position control of this arm was carried out. Three contractile PAMs are controlled to track a square signal, and the pressure of expansive PAM is set to $0.5 \mathrm{bar}$. The goal of this experiments is to test the position controllability of this arm. The reference signal for position of $L_{1}$ is a square signal of $590 \mathrm{~mm}-625 \mathrm{~mm}$ in amplitude, with a period of $20 \mathrm{~s}$, and the reference signal for position of $L_{2}$ and $L_{3}$ is a square signal of $600 \mathrm{~mm}-625 \mathrm{~mm}$ in amplitude, with a period of 20s. $L_{1}$ moves more than $L_{2}$ and $L_{3}$ which causes the arm to bend, so the arm is bending during the experiments. The control parameters of the FADRC and PID controller for the contractile PAMs are the same with the stiffness control experiments. The control results of position for the soft arm under no load and $500 \mathrm{~g}$ load are shown in figure 18 and figure 19.

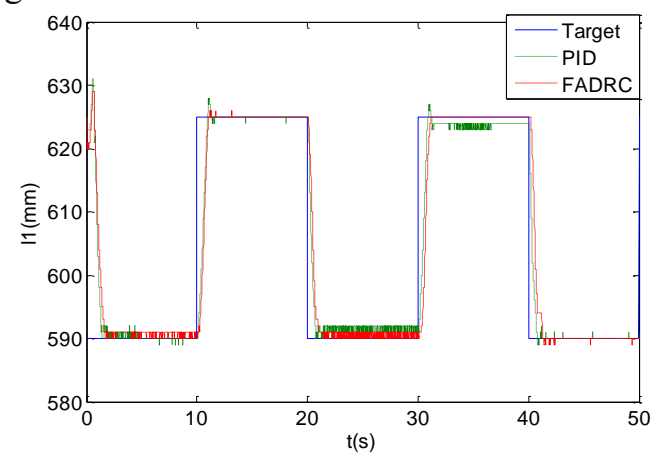

(a1)

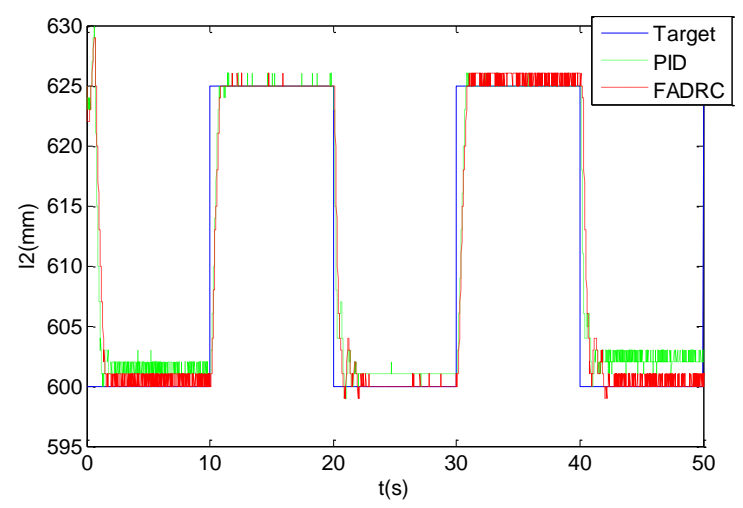

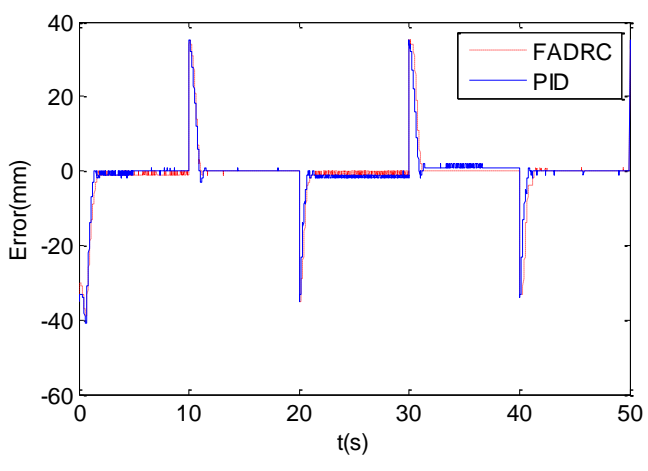

(a2)

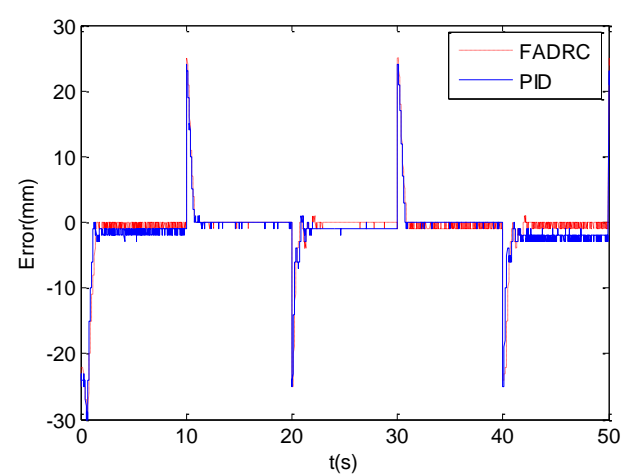


(b1)

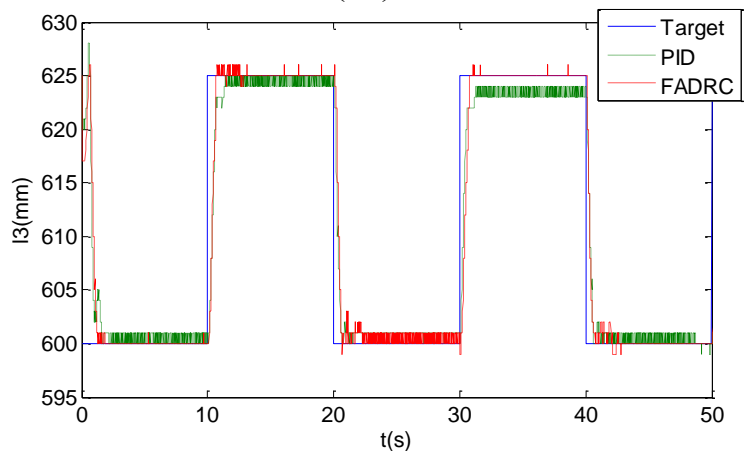

(c1) (b2)

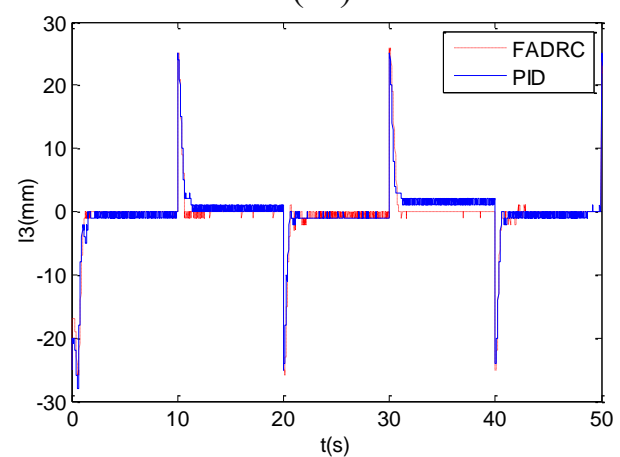

(c2)

Fig.18 Under no load, experiment results of position control of soft arm. (a1), (b1) and (c1) stand for the length of three contractile PAMs respectively. (a2), (b2), and(c2) are the corresponding error of (a1), (b1), and (c1).

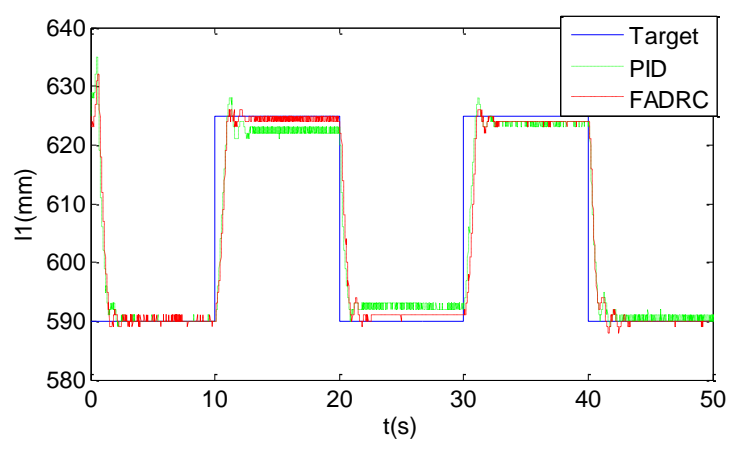

(a1)

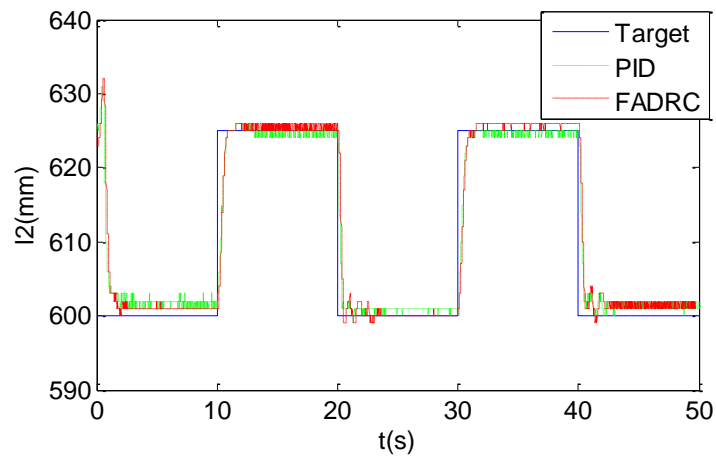

(b1)

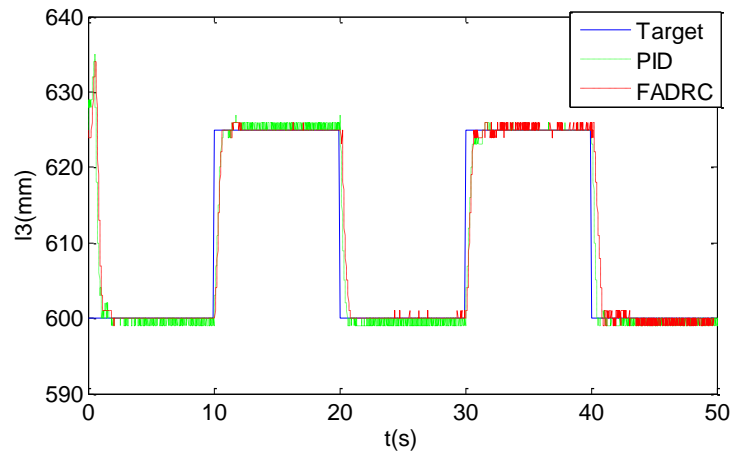

(c1)

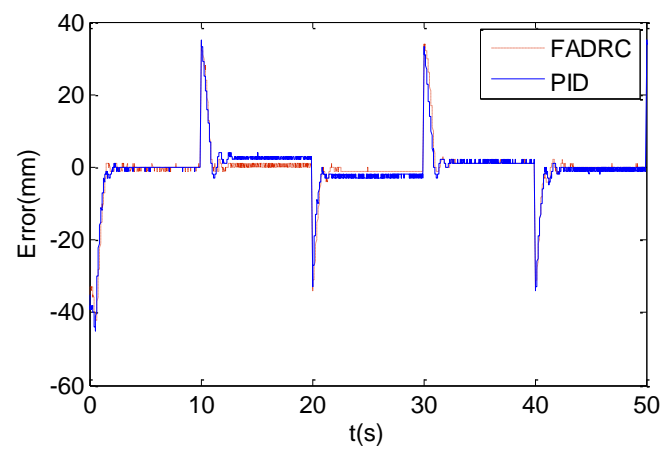

(a2)

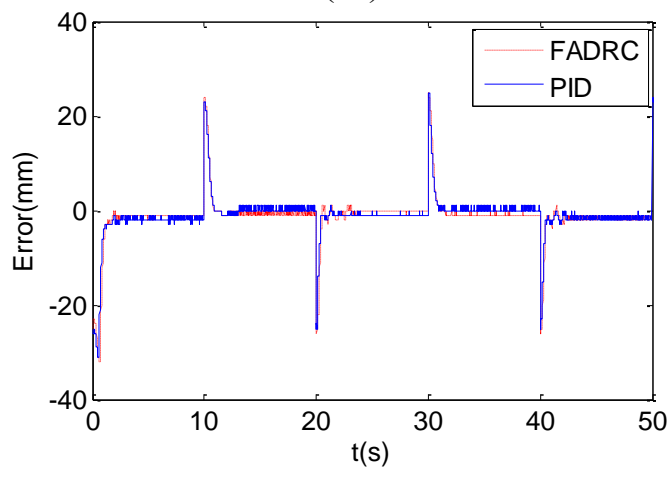

(b2)

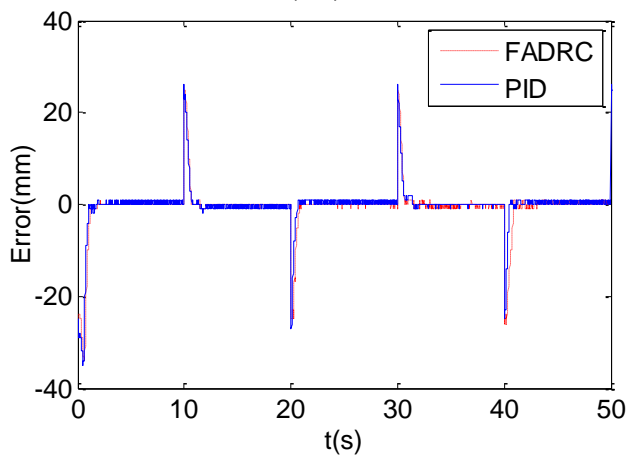

(c2)

Fig.19 Under 500g load, experiment results of position control of soft arm. (a1), (b1) and (c1) stand for the length of three contractile PAMs respectively. (a2), (b2), and(c2) are the corresponding error of (a1), (b1), and (c1).

Figure 18 and 19 are the position control results of PID and FADRC respectively. Based on figure 18, the maximum average error of PID and FADRC for $L_{1}, L_{2}$ and $L_{3}$ is $2.46 \mathrm{~mm}$ and $1.75 \mathrm{~mm}$, respectively. Based on Figure 19, the maximum average error rate of PID and FADRC for $L_{1}, L_{2}$ and $L_{3}$ is $3.32 \mathrm{~mm}$ and $2.85 \mathrm{~mm}$, respectively. From the position control results of the soft arm of figure 18 and 19, the soft arm possesses a relative good position controllability, and the feasibility of FADRC on the position control of soft arm is proved again. 


\section{Conclusion}

The innovation point of this new arm's design resides in allowing the variation of the arm's stiffness independently from its position in space. Compliance for the arm is desirable for tasks that require more flexibility and adaptability to the environment, and high stiffness characteristic is desirable for tasks that require for high position accuracy application. As argued in the introduction, light weight and soft is a very important characteristic for robot to be inherently safe for physical human-robot interaction. Thus, all these aspects are considered in the design of robot arm structures in the literature, however, in no other case the arm design combines a completely soft physical structure.

The pneumatically actuated soft arm possesses inherent light weight of and the ability to vary its stiffness independently of its length, and the control of a novel soft arm composed of three contractile PAMs and one expansive PAM which can vary its stiffness independent of its position in space, has been successfully presented. The stiffness models has been established in the paper. The paper demonstrates that the experimental data and data from the theoretical model both possess the same trend. When the arm is straight, there is a mean error of $4.09 \%$ and a maximum error of $5.46 \%$. After bending, the mean error is $4.30 \%$, while the maximum error rate is $6.33 \%$. A novel FADRC control method was proposed to implement the position and the variable stiffness control of the soft arm under no load and when carrying $500 \mathrm{~g}$ load, respectively. As compared to the PID controller, experiments demonstrated that the FADRC controller was much more effective in controlling the position and the stiffness. Future work will target a more precise stiffness model and kinematics model, and replicate the link described in this paper to allow the creation, model and control of a multilink manipulator.

\section{ACKNOWLEDGEMENTS}

This work was particularly supported by the National High Technology Research, Development Program of China (863 program) under Grant No. 2015AA042302, NSFC under grant 61573093, and the Fundamental Research Funds for the Central Universities of China under grant N150308001. The authors would also like to sincerely thank the reviewers and editors for their very pertinent remarks that helped this article become clearer and more precise.

\section{Reference}

[1]. Rongjie, K.,David, T. B.,Tianjiang, Z., etc. Design, modeling and control of a pneumatically actuated manipulator inspired by biological continuum structures[J]. Bioinspiration \& Biomimetics, 2013, 8 (3): 036008. [2]. Laschi, C.,Cianchetti, M.,Mazzolai, B., etc. Soft Robot Arm Inspired by the Octopus[J]. Advanced Robotics, 2012/01/01, 2012, 26 (7): 709-727.

[3]. McMahan, W.,Chitrakaran, V.,Csencsits, M., etc. Field trials and testing of the OctArm continuum manipulator[A]. In Proceedings 2006 IEEE International Conference on Robotics and Automation, 2006. ICRA 2006.[C], 15-19 May 2006, 2006; 2336-2341.

[4]. !!! INVALID CITATION !!! [4, 5].

[5]. Chen, G.,Pham, M. T.,Redarce, T. Sensor-based guidance control of a continuum robot for a semiautonomous colonoscopy[J]. Robotics and Autonomous Systems, 6/30/, 2009, 57 (6-7): 712-722.

[6]. G, F. A.,G, C. K. Bionic Handling Assistant[J]. Info-brochure, 2010.

[7]. Haddadin, S.,Albu-Schäffer, A.,Hirzinger, G. Requirements for Safe Robots: Measurements, Analysis and New Insights[J]. The International Journal of Robotics Research, November 1, 2009, 2009, 28 (11-12): $1507-$ 1527.

[8]. Vanderborght, B.,Albu-Schaeffer, A.,Bicchi, A., etc. Variable impedance actuators: a review[J]. Robotics and Autonomous Systems, 12/, 2013, 61 (12): 1601-14.

[9]. Grebenstein, M.,Albu-Schäffer, A.,Bahls, T., etc. The DLR hand arm system[A]. In 2011 IEEE International Conference on Robotics and Automation[C], 9-13 May 2011, 2011; 3175-3182.

[10]. Tonietti, G.,Schiavi, R.,Bicchi, A. Design and Control of a Variable Stiffness Actuator for Safe and Fast Physical Human/Robot Interaction[A]. In Proceedings of the 2005 IEEE International Conference on Robotics and Automation[C], 18-22 April 2005, 2005; 526-531.

[11]. Visser, L. C.,Carloni, R.,Stramigioli, S. Energy-Efficient Variable Stiffness Actuators[J]. IEEE Transactions on Robotics, 2011, 27 (5): 865-875.

[12]. Loeve, A. J.,van de Ven, O. S.,Vogel, J. G., etc. Vacuum packed particles as flexible endoscope guides with controllable rigidity[J]. Granular Matter, 12/, 2010, 12 (6): 543-54.

[13]. Brown, E.,Rodenberg, N.,Amend, J., etc. Universal robotic gripper based on the jamming of granular material[J]. Proceedings of the National Academy of Sciences of the United States of America, 11/02, 2010, 107 (44): 18809-14.

[14]. Rus, D.,Tolley, M. T. Design, fabrication and control of soft robots[J]. Nature, 05/28/print, 2015, 521 (7553): 467-475.

[15]. Jiang, A.,Xynogalas, G.,Dasgupta, P., etc. Design of a variable stiffness flexible manipulator with composite granular jamming and membrane coupling[A]. In 2012 IEEE/RSJ International Conference on Intelligent Robots and Systems (IROS 2012), 7-12 Oct. 2012[C], Piscataway, NJ, USA, 2012; 2922-7.

[16]. Cheng, N. G.,Lobovsky, M. B.,Keating, S. J., etc. Design and analysis of a robust, low-cost, highly 
articulated manipulator enabled by jamming of granular media[A]. In 2012 IEEE International Conference on Robotics and Automation, ICRA 2012[C], 2012; 4328-4333.

[17]. Xiang, C.,Giannaccini, M. E.,Theodoridis, T., etc. Variable stiffness Mckibben muscles with hydraulic and pneumatic operating modes[J]. Advanced Robotics, 2016/07/02, 2016, 30 (13): 889-899.

[18]. Tiwari, R.,Meller, M. A.,Wajcs, K. B., etc. Hydraulic artificial muscles[J]. Journal of Intelligent Material Systems and Structures, 02/, 2012, 23 (3): 301-12.

[19]. Galloway, K. C.,Becker, K. P.,Phillips, B., etc. Soft Robotic Grippers for Biological Sampling on Deep Reefs[J]. Soft Robotics, 2016, 3 (1): 23-33.

[20]. Ching-Ping, C.,Hannaford, B. Static and dynamic characteristics of McKibben pneumatic artificial muscles[A]. In Proceedings of the 1994 IEEE International Conference on Robotics and Automation[C], 8-13 May 1994, 1994; 281-286 vol.1.

[21]. Pritts, M. B.,Rahn, C. D. Design of an artificial muscle continuum robot[A]. In Robotics and Automation, 2004. Proceedings. ICRA '04. 2004 IEEE International Conference on[C], 26 April-1 May 2004, 2004; 47424746 Vol.5.

[22]. Suzumori, K.,Wakimoto, S.,Miyoshi, K., etc. Long bending rubber mechanism combined contracting and extending tluidic actuators[A]. In 2013 IEEE/RSJ International Conference on Intelligent Robots and Systems[C], 3-7 Nov. 2013, 2013; 4454-4459.

[23]. Han, J. From PID to Active Disturbance Rejection Control[J]. IEEE Transactions on Industrial Electronics, 2009, 56 (3): 900-906.

[24].jinqing, H. Active Disturbance Rejection Control Method[M]. National Defense Industry Press, 2008.

[25]. Yang, H.,Yu, Y.,Zhang, J. Angle tracking of a pneumatic muscle actuator mechanism under varying load conditions[J]. Control Engineering Practice, 4//, 2017, 61 1-10.

[26]. Zhao, L.,Li, Q.,Yang, H., etc. Positioning control of a one-DOF manipulator driven by pneumatic artificial muscles based on active disturbance rejection control[A]. In 2015 34th Chinese Control Conference (CCC)[C], 28-30 July 2015, 2015; 841-845.

[27]. Zhao, L.,Ge, L., Yang, Y. Active disturbance rejection trajectory tracking control for two-joint driven by pneumatic artificial muscles[A]. In 2015 International Conference on Fluid Power and Mechatronics (FPM)[C], 5-7 Aug. 2015, 2015; 972-977.

[28]. Gai, J. t.,Huang, S. d.,Huang, Q., etc. A new fuzzy active-disturbance rejection controller applied in PMSM position servo system[A]. In 2014 17th International Conference on Electrical Machines and Systems (ICEMS)[C], 22-25 Oct. 2014, 2014; 2055-2059.

[29]. Shijie, Y.,Xu, W. Active disturbance rejection fuzzy control of MTG fuel pressure[A]. In 2009 International Conference on Mechatronics and Automation[C], 9-12 Aug. 2009, 2009; 3015-3020.

[30]. QUANDong,QUANLi. The des ign and research of fuzzy self- adapted ADRC arithmetic[J]. China Academic Journal Electronic Publishing House, 2010, (5): 73-76.

[31]. Giannaccini, M. E.,Xiang, C. Q.,Atyabi, A., etc. Novel Design of a Soft Lightweight Pneumatic Continuum Robot Arm with Decoupled Variable Stiffness and Positioning[J]. Soft Robotics, 2016.

[32]. Neppalli, S.,Jones, B. A. Design, construction, and analysis of a continuum robot[A]. In 2007 IEEE/RSJ International Conference on Intelligent Robots and Systems[C], Oct. 29 2007-Nov. 2 2007, 2007; 1503-1507.

[33]. Jones, B. A.,Walker, I. D. Kinematics for multisection continuum robots[J]. IEEE Transactions on Robotics, 2006, 22 (1): 43-55.

[34]. Isuru, S. G.,Gustavo, A. M.-C.,David, T. B., etc. Modal kinematics for multisection continuum arms[J]. Bioinspiration \& Biomimetics, 2015, 10 (3): 035002.

[35]. Godage, I. S.,Branson, D. T.,Guglielmino, E., etc. Shape function-based kinematics and dynamics for variable length continuum robotic arms[A]. In 2011 IEEE International Conference on Robotics and Automation[C], 9-13 May 2011, 2011; 452-457.

[36]. Tsagarakis, N.,Caldwell, D. G.,Medrano-Cerda, G. A. A 7 DOF pneumatic muscle actuator (pMA) powered exoskeleton[A]. In Robot and Human Interaction, 1999. RO-MAN '99. 8th IEEE International Workshop on[C], 1999, 1999; 327-333. 